\title{
A study of differences between quark and gluon jets using vertex tagging of quark jets
}

\section{The OPAL Collaboration}

\begin{abstract}
Quark and gluon jets with equal energies are identified in three-jet hadronic $Z^{0}$ events, using reconstructed secondary vertices from heavy quark decay in conjunction with energy ordering of the jets to anti-tag the gluon jets. Selection of jets from a symmetric event topology allows their properties to be compared in a simple and direct manner. The jets under study have an energy of about $24 \mathrm{GeV}$. It is observed that gluon jets have a larger angular width than quark jets and yield a softer particle energy spectrum. Correspondingly, the mean particle multiplicity is found to be larger for gluon than for quark jets. Correcting the distributions for residual misidentification of the quark and gluon jets, the ratio of mean particle multiplicity of gluon relative to quark jets is measured to be

$$
\frac{\langle n\rangle_{\text {gluon }}}{\langle n\rangle_{\text {quark }}}=1.27 \pm 0.04 \text { (stat.) } \pm 0.06 \text { (syst.) }
$$

where the jets are defined using the $k_{\perp}$ jet finder. The numerical value of this ratio is found to be sensitive to the choice of the jet algorithm. The experimental results are compared to Monte Carlo calculations which incorporate perturbative QCD along with different assumptions about the hadronization process.
\end{abstract}

(Submitted to Zeitschrift für Physik C) 
P.D. Acton ${ }^{25}$, G. Alexander ${ }^{23}$, J. Allison ${ }^{16}$, P.P. Allport ${ }^{5}$, K.J. Anderson ${ }^{9}$, S. Arcelli ${ }^{2}$, A. Astbury $^{28}$, D. Axen ${ }^{29}$, G. Azuelos ${ }^{18, a}$, G.A.Bahan ${ }^{16}$, J.T.M.Baines ${ }^{16}$, A.H.Ball ${ }^{17}$, J. Banks ${ }^{16}$, R.J.Barlow ${ }^{16}$, S. Barnett ${ }^{16}$, J.R. Batley ${ }^{5}$, G. Beaudoin ${ }^{18}$, A. Beck ${ }^{23}$, G.A.Beck ${ }^{13}$, J.Becker ${ }^{10}$, T. Behnke ${ }^{27}$, K.W.Bell ${ }^{20}$, G. Bella ${ }^{23}$, P. Bentkowski ${ }^{18}$, P. Berlich ${ }^{10}$, S. Bethke ${ }^{11}$, O. Biebel ${ }^{3}$, U. Binder ${ }^{10}$, I.J. Bloodworth ${ }^{1}$, P. Bock ${ }^{11}$, B. Boden ${ }^{3}$, H.M. Bosch ${ }^{11}$, H. Breuker ${ }^{8}$, P. Bright-Thomas ${ }^{25}$, R.M. Brown ${ }^{20}$, A. Buijs ${ }^{8}$,

H.J. Burckhart ${ }^{8}$, C. Burgard ${ }^{27}$, P. Capiluppi ${ }^{2}$, R.K. Carnegie ${ }^{6}$, A.A.Carter ${ }^{13}$, J.R. Carter ${ }^{5}$, C.Y.Chang ${ }^{17}$, D.G. Charlton ${ }^{8}$, S.L. Chu ${ }^{4}$, P.E.L. Clarke ${ }^{25}$, I. Cohen ${ }^{23}$, J.C.Clayton ${ }^{1}$, W.J.Collins ${ }^{5}$, J.E.Conboy ${ }^{15}$, M.Cooper ${ }^{22}$, M. Coupland ${ }^{14}$, M. Cuffiani ${ }^{2}$, S. Dado ${ }^{22}$, G.M. Dallavalle ${ }^{2}$, S. De Jong ${ }^{13}$, L.A. del Pozo ${ }^{5}$, H. Deng ${ }^{17}$, A. Dieckmann ${ }^{11}$, M. Dittmar ${ }^{4}$, M.S. Dixit ${ }^{7}$, E. do Couto e Silva ${ }^{12}$, J.E. Duboscq ${ }^{8}$, E. Duchovni ${ }^{26}$, G. Duckeck ${ }^{11}$, I.P.Duerdoth ${ }^{16}$, D.J.P.Dumas ${ }^{6}$, P.A. Elcombe ${ }^{5}$, P.G. Estabrooks ${ }^{6}$, E. Etzion ${ }^{23}$, H.G. Evans ${ }^{9}$, F. Fabbri' ${ }^{2}$, M.Fierro ${ }^{2}$, M. Fincke-Keeler ${ }^{28}$, H.M.Fischer ${ }^{3}$, D.G. Fong ${ }^{17}$, M. Foucher ${ }^{17}$, A. Gaidot ${ }^{21}$, O. Ganel ${ }^{26}$, J.W. Gary ${ }^{4}$, J. Gascon ${ }^{18}$, R.F.McGowan ${ }^{16}$, N.I.Geddes ${ }^{20}$, C. Geich-Gimbel ${ }^{3}$, S.W.Gensler ${ }^{9}$, F.X.Gentit ${ }^{21}$, G. Giacomelli ${ }^{2}$,

R. Giacomelli ${ }^{2}$, V. Gibson ${ }^{5}$, W.R. Gibson ${ }^{13}$, J.D. Gillies ${ }^{20}$, J. Goldberg ${ }^{22}$, M.J. Goodrick ${ }^{5}$,

W. Gorn ${ }^{4}$, C. Grandi ${ }^{2}$, F.C.Grant ${ }^{5}$, J. Hagemann ${ }^{27}$, G.G. Hanson ${ }^{12}$, M. Hansroul ${ }^{8}$, C.K. Hargrove ${ }^{7}$, P.F. Harrison ${ }^{13}$, J. Hart ${ }^{8}$, P.M. Hattersley ${ }^{1}$, M. Hauschild ${ }^{8}$,

C.M.Hawkes ${ }^{8}$, E. Heflin ${ }^{4}$, R.J. Hemingway ${ }^{6}$, R.D. Heuer ${ }^{8}$, J.C. Hill ${ }^{5}$, S.J. Hillier ${ }^{8}$, T.Hilse ${ }^{10}$, D.A.Hinshaw ${ }^{18}$, J.D.Hobbs ${ }^{8}$, P.R. Hobson ${ }^{25}$, D. Hochman ${ }^{26}$, R.J. Homer ${ }^{1}$, A.K. Honma ${ }^{28, a}$, R.E. Hughes- Jones ${ }^{16}$, R. Humbert ${ }^{10}$, P. Igo-Kemenes ${ }^{11}$, H. Ihssen ${ }^{11}$, D.C.Imrie ${ }^{25}$, A.C.Janissen ${ }^{6}$, A. Jawahery ${ }^{17}$, P.W. Jeffreys ${ }^{20}$, H. Jeremie ${ }^{18}$, M. Jimack ${ }^{2}$, M. Jobes ${ }^{1}$, R.W.L. Jones ${ }^{13}$, P. Jovanovic ${ }^{1}$, C. Jui ${ }^{4}$, D. Karlen ${ }^{6}$, K. Kawagoe ${ }^{24}$, T. Kawamoto ${ }^{24}$, R.K. Keeler ${ }^{28}$, R.G. Kellogg ${ }^{17}$, B.W. Kennedy ${ }^{15}$, S. Kluth ${ }^{5}$,

T. Kobayashi ${ }^{24}$, D.S. Koetke ${ }^{8}$, T.P. Kokott ${ }^{3}$, S. Komamiya ${ }^{24}$, L. Köpke ${ }^{8}$, J.F. Kral ${ }^{8}$, R. Kowalewski ${ }^{6}$, J.von $\mathrm{Krogh}^{11}$, J. Kroll ${ }^{9}$, M. Kuwano ${ }^{24}$, P. Kyberd ${ }^{13}$, G.D. Lafferty ${ }^{16}$, R. Lahmann ${ }^{17}$, F. Lamarche ${ }^{18}$, J.G. Layter ${ }^{4}$, P. Leblanc ${ }^{18}$, A.M. Lee ${ }^{17}$, M.H. Lehto ${ }^{15}$, D. Lellouch ${ }^{26}$, C. Leroy $^{18}$, J. Letts ${ }^{4}$, S. Levegrün ${ }^{3}$, L. Levinson ${ }^{26}$, S.L. Lloyd ${ }^{13}$, F.K. Loebinger ${ }^{16}$, J.M. Lorah ${ }^{17}$, B. Lorazo ${ }^{18}$, M.J. Losty ${ }^{7}$, X.C. Lou ${ }^{12}$, J. Ludwig ${ }^{10}$, M. Mannelli ${ }^{8}$, S. Marcellini ${ }^{2}$, G. Maringer ${ }^{3}$, C. Markus ${ }^{3}$, A.J. Martin ${ }^{13}$, J.P. Martin ${ }^{18}$, T. Mashimo ${ }^{24}$, P. Mättig ${ }^{3}$, U.Maur ${ }^{3}$, J. McKenna ${ }^{28}$, T.J. McMahon ${ }^{1}$, J.R. McNutt ${ }^{25}$, F. Meijers ${ }^{8}$, D. Menszner ${ }^{11}$, F.S. Merritt ${ }^{9}$, H. Mes ${ }^{7}$, A. Michelini ${ }^{8}$, R.P. Middleton ${ }^{20}$, G. Mikenberg ${ }^{26}$, J. Mildenberger ${ }^{6}$, D.J. Miller ${ }^{15}$, R. Mir ${ }^{12}$, W. Mohr ${ }^{10}$, C. Moisan ${ }^{18}$, A. Montanari ${ }^{2}$, T. Mori ${ }^{24}$, M. Morii ${ }^{24}$, T. Mouthuy ${ }^{12, b}$, B. Nellen ${ }^{3}$, H.H. Nguyen ${ }^{9}$, M. Nozaki ${ }^{24}$, S.W.O'Neale ${ }^{1}$, F.G.Oakham ${ }^{7}$, F.Odorici ${ }^{2}$, H.O.Ogren ${ }^{12}$, C.J.Oram ${ }^{28, a}$, M.J. Oreglia ${ }^{9}$, S. Orito ${ }^{24}$, J.P.Pansartt ${ }^{21}$, B. Panzer-Steindel ${ }^{8}$, P. Paschievici ${ }^{26}$, G.N.Patrick ${ }^{20}$, N.Paz-Jaoshvili2i ${ }^{23}$, P.Pfister ${ }^{10}$, J.E. Pilcher ${ }^{9}$, J. Pinfold ${ }^{31}$, D. Pitman ${ }^{28}$, D.E.Plane ${ }^{8}$, P. Poffenberger ${ }^{28}$, B. Poli ${ }^{2}$, A. Pouladdej ${ }^{6}$, T.W.Pritchard ${ }^{13}$, H. Przysiezniak ${ }^{18}$, G. Quast ${ }^{27}$, M.W. Redmond ${ }^{9}$, D.L. Rees $^{8}$, G.E. Richards ${ }^{16}$, D. Robinson ${ }^{8}$, A. Rollnik ${ }^{3}$, J.M. Roney ${ }^{28, c}$, E. Ros $^{8}$, S. Rossberg $^{10}$, A.M. Rossi ${ }^{2}$, M. Rosvick ${ }^{28}$, P. Routenburg ${ }^{6}$, K. Runge ${ }^{10}$, O. Runolfsson ${ }^{8}$, D.R.Rust ${ }^{12}$, M.Sasaki ${ }^{24}$, C.Sbarra ${ }^{8}$, A.D.Schaile ${ }^{10}$, O.Schaile ${ }^{10}$, W.Schappert ${ }^{6}$, P.Scharff-Hansen ${ }^{8}$, P. Schenk ${ }^{4}$, B.Schmitt ${ }^{3}$, H. von der Schmitt ${ }^{11}$, S.Schreiber ${ }^{3}$, C.Schwick ${ }^{27}$, J.Schwiening ${ }^{3}$,

W.G.Scott ${ }^{20}$, M.Settles ${ }^{12}$, T.G.Shears ${ }^{5}$, B.C.Shen ${ }^{4}$, C.H.Shepherd-Themistocleous ${ }^{7}$, P.Sherwood ${ }^{15}$, R.Shypit ${ }^{29}$, A.Simon ${ }^{3}$, P.Singh ${ }^{13}$, G.P.Siroli ${ }^{2}$, A.Skuja ${ }^{17}$, A.M.Smith ${ }^{8}$, T.J.Smith ${ }^{28}$, G.A.Snow ${ }^{17}$, R.Sobie ${ }^{28, c}$, R.W.Springer ${ }^{17}$, M.Sproston ${ }^{20}$, K. Stephens ${ }^{16}$, J.Steuerer ${ }^{28}$, R.Ströhmer ${ }^{11}$, D.Strom ${ }^{30}$, T. Takeshita ${ }^{24, d}$, P. Taras ${ }^{18}$, S. Tarem ${ }^{26}$, M. Tecchio ${ }^{9}$, P. Teixeira-Dias ${ }^{11}$, N. Tesch ${ }^{3}$, N.J. Thackray ${ }^{1}$, M.A.Thomson ${ }^{15}$, 
E. Torrente-Lujan ${ }^{22}$, G. Transtromer ${ }^{25}$, N.J. Tresilian ${ }^{16}$, T. Tsukamoto ${ }^{24}$, M.F. Turner ${ }^{8}$, G. Tysarczyk-Niemeyer ${ }^{11}$, D. Van den plas ${ }^{18}$, R. Van Kooten ${ }^{27}$, G.J. VanDalen ${ }^{4}$, G. Vasseur ${ }^{21}$, C.J. Virtue ${ }^{7}$, A. Wagner ${ }^{27}$, D.L.Wagner ${ }^{9}$, C. Wahl ${ }^{10}$, J.P. Walker ${ }^{1}$, C.P. Ward ${ }^{5}$, D.R. Ward ${ }^{5}$, P.M. Watkins ${ }^{1}$, A.T. Watson ${ }^{1}$, N.K. Watson ${ }^{8}$, M. Weber ${ }^{11}$, P. Weber ${ }^{6}$, P.S. Wells ${ }^{8}$, N. Wermes ${ }^{3}$, M.A. Whalley ${ }^{1}$, G.W.Wilson ${ }^{4}$, J.A. Wilson ${ }^{1}$, V-H. Winterer ${ }^{10}$, T. Wlodek ${ }^{26}$, S. Wotton ${ }^{11}$, T.R. Wyatt $^{16}$, R. Yaari ${ }^{26}$, A. Yeaman ${ }^{13}$, G. Yekutieli ${ }^{26}$, M. Yurko ${ }^{18}$, W.Zeuner ${ }^{8}$, G.T.Zorn ${ }^{17}$.

\footnotetext{
${ }^{1}$ School of Physics and Space Research, University of Birmingham, Birmingham, B15 2TT, UK
}

${ }^{2}$ Dipartimento di Fisica dell' Università di Bologna and INFN, Bologna, 40126, Italy

${ }^{3}$ Physikalisches Institut, Universität Bonn, D-5300 Bonn 1, FRG

${ }^{4}$ Department of Physics, University of California, Riverside, CA 92521 USA

${ }^{5}$ Cavendish Laboratory, Cambridge, CB3 0HE, UK

${ }^{6}$ Carleton University, Dept of Physics, Colonel By Drive, Ottawa, Ontario K1S 5B6, Canada

${ }^{7}$ Centre for Research in Particle Physics, Carleton University, Ottawa, Ontario K1S 5B6, Canada

${ }^{8}$ CERN, European Organisation for Particle Physics, 1211 Geneva 23, Switzerland

${ }^{9}$ Enrico Fermi Institute and Department of Physics, University of Chicago, Chicago Mlinois 60637 , USA

${ }^{10}$ Fakultät für Physik, Albert Ludwigs Universität, D-7800 Freiburg, FRG

${ }^{11}$ Physikalisches Institut, Universität Heidelberg, Heidelberg, FRG

${ }^{12}$ Indiana University, Dept of Physics, Swain Hall West 117, Bloomington, Indiana 47405, USA

${ }^{13}$ Queen Mary and Westfield College, University of London, London, E1 4NS, UK

${ }^{14}$ Birkbeck College, London, WC1E 7HV, UK

${ }^{15}$ University College London, London, WC1E 6BT, UK

${ }^{16}$ Department of Physics, Schuster Laboratory, The University, Manchester, M13 9PL, UK

${ }^{17}$ Department of Physics, University of Maryland, College Park, Maryland 20742, USA

${ }^{18}$ Laboratoire de Physique Nucléaire, Université de Montréal, Montréal, Quebec, H3C 3J7, Canada

${ }^{20}$ Rutherford Appleton Laboratory, Chilton, Didcot, Oxfordshire, OX11 0QX, UK

${ }^{21}$ DAPNIA/SPP, Saclay, F-91191 Gif-sur-Yvette, France

${ }^{22}$ Department of Physics, Technion-Israel Institute of Technology, Haifa 32000, Israel

${ }^{23}$ Department of Physics and Astronomy, Tel Aviv University, Tel Aviv 69978, Israel

${ }^{24}$ International Centre for Elementary Particle Physics and Dept of Physics, University of Tokyo, Tokyo 113, and Kobe University, Kobe 657, Japan

${ }^{25}$ Brunel University, Uxbridge, Middlesex, UB8 3PH UK

${ }^{26}$ Nuclear Physics Department, Weizmann Institute of Science, Rehovot, 76100, Israel

${ }^{27}$ Universität Hamburg/DESY, II Inst für Experimental Physik, 2000 Hamburg 52, Germany

${ }^{28}$ University of Victoria, Dept of Physics, P O Box 3055, Victoria BC V8W 3P6, Canada ${ }^{29}$ University of British Columbia, Dept of Physics, 6224 Agriculture Road, Vancouver BC V6T 1Z1, Canada 
${ }^{30}$ University of Oregon, Dept of Physics, Eugene, Oregon 97403, USA

${ }^{31}$ University of Alberta, Dept of Physics, Edmonton AB T6G 2J1, Canada

${ }^{a}$ Also at TRIUMF, Vancouver, Canada V6T $2 \mathrm{~A} 3$

${ }^{b}$ Now at Centre de Physique des Particules de Marseille, Faculté des Sciences de Luminy, Marseille

${ }^{c}$ And IPP, University of Victoria, Dept of Physics, P O Box 3055, Victoria BC V8W 3P6, Canada

${ }^{d}$ Also at Shinshu University, Matsumoto 390, Japan 


\section{Introduction}

The question of whether jets initiated by a highly virtual quark or gluon possess different properties has been the object of considerable theoretical [1]-[4] and experimental [5]-[6] study. In Quantum Chromodynamics (QCD), the gluon is associated with a color charge $\mathrm{C}_{A}=3$ and the quark with a charge $\mathrm{C}_{F}=4 / 3$ and one naïvely expects a ratio of $\left(\mathrm{C}_{A} / \mathrm{C}_{F}\right)=9 / 4$ for the multiplicity of soft gluons produced from the two jet types [1]. For equal quark and gluon jet energies, the larger multiplicity of the gluon jet means that its particle energy spectrum is softer. This in turn implies that the angles of particles relative to the jet axis should be larger in gluon than in quark jets of equal energy, because the mean transverse energy value of the particles is expected to be about the same: thus that gluon jets are spread over a larger angular interval than quark jets and in this sense are broader. These predictions are valid at leading order and assume that the jet energies are large enough that the numerical results become independent of the jet energy. A number of theoretical studies suggests, however, that large non-leading and finite energy corrections significantly reduce the magnitude of the differences expected between quark and gluon jets $[3,4]$. Furthermore, to relate the predictions to the hadron-level measurements, it is necessary to employ a hadronization model or else to invoke an ansatz like local parton-hadron duality [7]. The situation has also been confused experimentally because of the use of quark and gluon jets with different energies and because the quark jets have sometimes been taken from two-jet events in $\mathrm{e}^{+} \mathrm{e}^{-}$annihilations while the gluon jets are taken from three-jet events, whereas model calculations imply that the jet energy and environment substantially influence quark and gluon jet properties. As a consequence, the experimental results have sometimes been contradictory and inconclusive, being dependent on QCD Monte Carlos for interpretation. It has therefore proven difficult to establish whether jets of hadrons initiated by quarks and gluons possess different characteristics and the level to which such differences are expected.

In a previous publication [6], we addressed the question of quark and gluon jet differences from an experimental standpoint, using the jet identification technique which we introduced in [8]. The purpose of this analysis was to investigate whether differences between quark and gluon jets were observable from our data. Symmetric three-jet events were employed, in which the angles between the highest energy jet and each of the two lower energy jets were the same. For the configuration chosen, the highest energy jet was known to be a quark or an antiquark jet with high probability, because of the bremsstrahlung nature of gluon radiation. A high energy lepton, assumed to originate from charm or bottom quark semi-leptonic decay, was required to be present in one of the two lower energy jets. Since heavy quarks in $\mathrm{e}^{+} \mathrm{e}^{-}$annihilation are produced predominantly at the electro-weak vertex, the observation of such a lepton identified the jet as being a quark jet, with high probability. The lower energy jet without the lepton was thus "anti-tagged" as the gluon jet. To obtain an unbiased sample of quark jets with which to compare 
these gluon jets, the same symmetric event sample was employed, but without the lepton tag requirement. In this case, the two lower energy jets were known to be an equal mixture of quark and gluon jets and were presumed to have normal properties: comparing these jets to the anti-tagged gluon jets from the tagged events allowed a comparison of quark and gluon jet properties because the latter sample had a much larger gluon jet component. Since the quark and gluon jets being compared had the same energy, event environment and selection criteria, they could be compared directly, with no need for Monte Carlo calculations to establish the results. It was observed that gluon jets were broader and yielded a softer particle energy spectrum than quark jets, in qualitative agreement with the expectations of perturbative QCD. Gluon jets were observed to have a slightly larger mean particle multiplicity than quark jets.

In this publication, we update our previous study using a data sample which is about seven times larger. The data were collected using the OPAL detector at the $\mathrm{e}^{+} \mathrm{e}^{-}$collider LEP at CERN. We introduce a new technique to anti-tag the gluon jet, based on reconstructed secondary vertices to identify bottom quark jets instead of high energy leptons. Because of their relatively long lifetime $\left(\tau \sim 10^{-12} \mathrm{~s}\right)$, bottom hadrons travel a considerable distance from their point of creation before they decay. They thus lead to "secondary vertices" displaced from the primary $\mathrm{e}^{+} \mathrm{e}^{-}$collision point, from which the bottom hadron decay products emanate. Reconstruction of secondary vertices in jets therefore permits bottom quark jets to be identified and the methods we developed in our earlier study of symmetric three jet events to be applied. Furthermore, the tagged event sample from secondary vertices has little overlap with the lepton tagged one and so provides a consistency check on our previous results. In our previous study, the quark and gluon jets were not corrected for residual quark and gluon jet misidentification. Here, we evaluate the purity of the identified quark and gluon jets using Monte Carlo. After presenting the data directly in a model independent manner, we correct the measurements for the residual quark-gluon jet impurities. The results are then compared to QCD models containing different assumptions about the perturbative phase and the hadronization process.

\section{Detector and data sample}

The OPAL detector has been described in detail elsewhere [9]. Only a brief account of some relevant features for the present analysis and of the selection of hadronic $Z^{0}$ decays is given here.

The tracking of charged particles is performed with a central detector, which contains three systems of drift chambers: a precision inner vertex chamber, a large volume jet chamber and specialized chambers at the outer radius of the jet chamber 
which improve the measurements in the $z$-direction. ${ }^{1}$ The OPAL central detector also includes a silicon microvertex detector which is discussed in the next paragraph. The tracking chambers are enclosed by a solenoidal magnet coil providing an axial field of approximately $0.435 \mathrm{~T}$. The most important tracking detector for the momentum measurement is the jet chamber, which provides up to 159 space-points per track and close to $100 \%$ track finding efficiency for charged tracks in the region $|\cos \theta|<0.92$. The momentum resolution for charged tracks is $\Delta p / p \sim 5 \%$ for $p \sim 45 \mathrm{GeV} / c$. The average angular resolution is about $0.1 \mathrm{mrad}$ in $\phi$ and better than $10 \mathrm{mrad}$ in $\theta$.

Of particular importance to this analysis is the silicon microvertex detector [10], installed as a part of OPAL during the 1990-1991 LEP shutdown. This device consists of two layers of silicon microstrip detectors positioned close to the $\mathrm{e}^{+} \mathrm{e}^{-}$ collision point, one at a radius of $6.1 \mathrm{~cm}$ with an angular coverage of $|\cos \theta|<0.83$ and one at a radius of $7.5 \mathrm{~cm}$ with a coverage of $|\cos \theta|<0.77$. We presently achieve a positional resolution of about $10 \mu \mathrm{m}$ with this detector and an efficiency of about $95 \%$ for finding at least one silicon detector hit on a track, for tracks which are reconstructed in multihadronic events in the other tracking chambers and which pass through the active silicon region.

Electromagnetic energy is measured by a detector composed of lead-glass blocks located outside the magnet coil, with a barrel $(|\cos \theta|<0.82)$ and two endcap $(0.81<|\cos \theta|<0.98)$ parts. Each block subtends approximately $40 \times 40 \mathrm{mrad}^{2}$. The depth of material to the back of the calorimeter is about 25 radiation lengths. The basic calorimeter entities used are clusters of energy, i.e. groups of contiguous blocks containing a non-negligible energy deposition from the traversing particles. To minimize double counting of energy, clusters are accepted only if they are unassociated with a charged track. A cluster is associated with a charged track if the extrapolated track coordinates at the entrance of the calorimeter match to better than $80 \mathrm{mrad}$ in $\phi$ and $150 \mathrm{mrad}$ in $\theta$, if the cluster is in the barrel, or $50 \mathrm{mrad}$ in both $\phi$ and $\theta$, if it is in the endcap.

For muon identification, OPAL is instrumented with a hadron calorimeter, located outside the electromagnetic one, constructed from alternating layers of iron slabs and limited streamer tubes. The depth of the material is typically eight or more interaction lengths. Outside the hadron calorimeter is the muon chamber system, comprised of four layers of drift chambers for $|\cos \theta|<0.68$ and four layers of limited streamer tubes for $0.60<|\cos \theta|<0.98$. The typical positional resolution of the muon chambers is about $2 \mathrm{~mm}$.

The OPAL trigger system is described in [11] and the online selection procedures for hadronic events in [12]. Within the geometrical region used for the present study, the efficiency of this selection is greater than $99.6 \%$. Tracks and clusters used in

\footnotetext{
${ }^{1}$ Our coordinate system is defined so that $z$ is the coordinate parallel to the $\mathrm{e}^{+}$and $\mathrm{e}^{-}$beam axis, $r$ is the coordinate normal to the beam axis, $\phi$ is the azimuthal angle and $\theta$ is the polar angle with respect to $z$.
} 
this analysis were subjected to quality cuts. Charged tracks were required to have at least 20 measured points in the jet chamber, to have a transverse momentum in the $r$ - $\phi$ plane greater than $0.15 \mathrm{GeV} / c$, to lie in the region $|\cos \theta|<0.94$ and to point to the origin to within $5 \mathrm{~cm}$ in the $r$ - $\phi$ plane. In addition, they were required to yield a $\chi^{2}$ per degree-of-freedom of less than 100 for the track fit in the $r$ - $\phi$ plane. Besides the requirement that they be unassociated with charged tracks, clusters were required to have an energy greater than $0.1 \mathrm{GeV}$ if they were in the barrel part of the detector and to be spread over at least two lead glass blocks and have an energy greater than $0.3 \mathrm{GeV}$ if they were in the endcap part. For the present analysis, particles are defined to be one of these accepted charged tracks or unassociated clusters.

Additional event cuts were applied to eliminate residual background and to obtain a data sample of good quality. The thrust axis of the event was calculated using the particles, and was required to satisfy $\left|\cos \left(\theta_{\text {thrust }}\right)\right|<0.9$, to eliminate events where a significant number of particles were lost or badly measured near the beam direction. The number of accepted charged tracks was required to be at least five to eliminate $\tau^{+} \tau^{-}$events. We required the calorimeters and tracking chambers to be fully operational and restricted the analysis to our 1991 and 1992 data, since these were collected after the silicon microvertex system had been installed. Only those events collected within $100 \mathrm{MeV}$ of the $Z^{0}$ peak were used. The total integrated luminosity employed was about $33 \mathrm{pb}^{-1}$, corresponding to an initial sample of 1,003,140 hadronic $Z^{0}$ decays.

\section{$3 \quad$ Three-jet event selection and jet tagging}

Three-jet events were selected by applying the $k_{\perp}$, or "Durham", jet finder [13] to the accepted particles. We choose this jet finder because it is well defined in perturbation theory, allowing calculations to incorporate leading terms to all orders. It is therefore expected to facilitate comparison between theory and the results presented in this publication. Briefly, pairs of resolvable jets within an event were required to satisfy

$$
y_{i j} \equiv \frac{2 \cdot \min \left(E_{i}^{2}, E_{j}^{2}\right) \cdot\left(1-\cos \theta_{i j}\right)}{E_{\text {vis. }}^{2}}>y_{\text {cut }},
$$

where $E_{i}$ and $E_{j}$ are the energies of two jets or particles $i$ and $j$ and $\theta_{i j}$ is the angle between them. The visible energy of the event, $E_{\text {vis., }}$ was obtained by summing the energies of the particles. In this analysis, the energy values of charged tracks are calculated by assigning them the pion mass, while clusters are assumed to be photons. The jet directions are equal to the vector sum of the constituent particle three-momenta. Jets or particles with $y_{i j} \leq y_{\text {cut }}$ were combined into a single jet [13]. The value $y_{c u t}=0.02$ was chosen because this yields a maximum number of threejet events for the angular configuration which we study. Each jet was required to contain at least two particles, lie in the polar angle region $|\cos \theta|<0.9$ and 
have a visible energy, $E_{\text {vis. }}^{\text {jet }}$, larger than $5 \mathrm{GeV}$, where $E_{\text {vis. }}^{\text {jet }}$ is defined by summing the energies of the particles assigned to the jets. The sum of the angles between the three jets was required to exceed $358^{\circ}$ to eliminate non-planar events. The jets were assigned a calculated energy using the angles between them, assuming massless kinematics and perfect event reconstruction. Symmetric three-jet events were selected by projecting the jets into the three-jet event plane and requiring that the angle between the highest energy jet and the two others be in the range $150 \pm$ $10^{\circ}$. The event plane is defined by the plane normal to the smallest sphericity [14] eigenvector. In total, 22,637 symmetric three-jet events were obtained. Assuming the flavor independence of the strong coupling constant [15], it may be presumed that these events approximately represent a normal mixture of quark flavors and decays at the $Z^{0}$ peak. We refer to them in the following as the normal-mixture sample. The mean calculated jet energies, the mean visible jet energies and the mean angles between the jets are summarized in table 1 for these data.

Finite quark mass values lead to a reduction in the phase space available for gluon emission in heavy quark events compared to light quark ones. The relative population of bottom quark events is, as a consequence, expected to be a few percent smaller in the symmetric three-jet event sample than it is in the inclusive multihadron one, even assuming the flavor independence of the strong coupling constant. Thus the symmetric three jet sample discussed in this analysis is expected to differ slightly from a true normal mixture of quark flavors at the $Z^{0}$ peak. We nonetheless retain the label "normal-mixture" for these data, for reasons of convenience.

Our analysis technique relies on anti-tagging the gluon jet in the symmetric threejet sample, as explained in the introduction. For this, we required a reconstructed secondary vertex to be present in one of the two lower energy jets. The secondary vertex is associated with heavy quark decay: thus, the gluon jet was assumed to be the other of the two lower energy jets. In order to define secondary vertices, it was first necessary to determine the $\mathrm{e}^{+} \mathrm{e}^{-}$collision point. The $\mathrm{e}^{+} \mathrm{e}^{-}$collision point was determined for each event using a beam constrained primary vertex fit. Briefly, tracks which passed within $2 \mathrm{~cm}$ of the mean $\mathrm{e}^{+} \mathrm{e}^{-}$collision position in the $r$ - $\phi$ plane were constrained to a primary event vertex; tracks were removed from the fit if they contributed a $\chi^{2}$ value larger than 4.0 and the remaining tracks were refitted. The procedure was iterated until all tracks in the fit contributed a $\chi^{2}$ value smaller than 4.0. The method used to obtain the mean $\mathrm{e}^{+} \mathrm{e}^{-}$collision position is described in [16]. Secondary vertices were identified inside a jet using the following procedure. Tracks in the jet were selected if they contained at least one silicon microvertex detector hit, in addition to the criteria listed in section 2. The assignment of a particle to a jet was obtained from the jet finder. The tracks were required to have a distance of closest approach to the $\mathrm{e}^{+} \mathrm{e}^{-}$collision point of less than $0.3 \mathrm{~cm}$ in the $r$ - $\phi$ plane and an error on that quantity of less than $0.1 \mathrm{~cm}$. All tracks in the jet fulfilling these criteria were fitted to a common secondary vertex in the $r-\phi$ plane. Similarly to the primary vertex fit, the track with the largest contribution to the $\chi^{2}$ of the fit was removed if its $\chi^{2}$ contribution was larger than 4.0. The 
remaining tracks were then refitted and the procedure iterated until all tracks in the fit contributed a $\chi^{2}$ value less than 4.0 or else there were fewer than three tracks remaining, in which case the fit was deemed to have failed.

For jets with a successful fit, a decay length was defined to be the distance in the $r$ - $\phi$ plane between the reconstructed secondary vertex position and the $\mathrm{e}^{+} \mathrm{e}^{-}$ collision point. The sign of the decay length was determined by summing the threemomenta of the tracks fitted to the secondary vertex. If this vector was in the same hemisphere as the vector between the beam collision point and the secondary vertex position, the decay length was positive, otherwise it was negative. The event was retained as a tagged event if one of the two lower energy jets had a secondary vertex with a positive decay length between 0.15 and $0.50 \mathrm{~cm}$, while the other one did not have a secondary vertex or else its decay length was less than $0.15 \mathrm{~cm}$. In total, 1175 tagged events were obtained: in the following, we refer to these as the tagged sample. We refer to the lower energy jet without the secondary vertex tag as the gluon-tagged jet. The tagged sample is therefore a subset of the normal-mixture one discussed above, but since it is a small subset we assume the two data sets to be statistically independent. The mean jet energies and angles between the jet directions are listed in table 1 for the tagged sample. It is seen that the energies of the gluon-tagged jets are the same as those of the lower energy jets in the normalmixture sample to better than $2 \%$ : the corresponding mean difference in angle with respect to the highest energy jets is only $0.7^{\circ}$.

In fig. 1 (a), we show the decay length distributions of the two lower energy jets of the normal-mixture sample, added together. The distribution is seen to be asymmetric, with more entries at positive values of decay length than at negative values. The histogram shows the Monte Carlo prediction from the Jetset parton shower model [18], using the Peterson fragmentation function [25] for heavy quark production. Our implementation of this model is presented in section 4.1. The Monte Carlo sample includes simulation of the detector [17] and the same selection criteria as the data. ${ }^{2}$ The model is seen to provide a good description of the measurements. The contributions to the decay length distribution from bottom quark, charm quark, light quark and gluon jets are shown separately for the simulated events by the shaded curves. The excess of entries at positive values of decay length is seen to arise principally from the long-lived bottom quarks. The decay length interval from 0.15 to $0.50 \mathrm{~cm}$ used to tag quark jets is delineated by the vertical dashed lines in fig. 1 (a). Figure 1 (b) shows an enlargement around this region. By selecting jets with large decay lengths, we preferentially obtain bottom quark events, as intended, since this yields an efficient discrimination against the gluon jets.

\footnotetext{
${ }^{2}$ In addition, some rescaling of the track parameters is applied to the Monte Carlo in order to improve the agreement with the data.
} 


\section{$4 \quad$ Estimated quark and gluon jet purities}

To evaluate the purity of the quark and gluon jets in the normal-mixture and tagged samples, we use Monte Carlo events which include full simulation of the OPAL detector [17] and the same event selection and analysis procedures as the data. ${ }^{2}$ These estimated quark and gluon jet purities are purely informational for the direct, model independent comparisons presented in section 5. They are used in section 6 , however, to correct the data for the residual quark-gluon jet misidentification. Below, we discuss the Monte Carlo models used in our analysis and their results for jet purity. Following this, we present distributions from the data which substantiate the purity values obtained from the Monte Carlo.

\subsection{Monte Carlo models and jet purities}

For the underlying QCD physics event generators, we use Jetset, version 7.3 [18], Herwig, version 5.5 [19], and Cojets, versions 6.12 and 6.23 [20]. In these generators, the quarks and gluons created in $Z^{0}$ decay evolve through a parton shower to low mass values, followed by string, cluster and independent hadronization for Jetset, Herwig and Cojets, respectively. The parameter values of the models have been tuned to provide a good representation of the global event characteristics of hadronic $Z^{0}$ decays: Jetset and Herwig by OPAL and Cojets by its authors [22]. The OPAL parameter values for Jetset are given in [21]. Our tuned values for Herwig55 are listed in table 2. ${ }^{3}$ However, Jetset with its standard hadronization mechanism is known to yield too hard an energy spectrum for bottom hadrons [23], while the bottom hadron energy spectrum from Herwig is, in comparison, very soft [24]. In this sense, Jetset and Herwig present opposite extremes as models for bottom hadron production. We also use Jetset in a non-standard mode, for which charm and bottom quark hadronization are described by the fragmentation function of Peterson et al. [25], in which case Jetset is known to describe well the available bottom hadron energy measurements from LEP [26]. For the variant of Jetset which uses Peterson fragmentation, the parameter values were tuned by us to simultaneously describe OPAL measurements of global event properties in events with and without a hard, identified lepton associated with semi-leptonic bottom quark decay; the resulting values are given in table 3 .

The mass cutoff which terminates shower evolution is considerably larger in Cojets $\left(3 \mathrm{GeV} / c^{2}\right)$ than it is in Jetset or Herwig (about $1 \mathrm{GeV} / c^{2}$ ). This means that differences between quark and gluon jets at the parton level, related to the different color charges of quarks and gluons, are less important in the former case. For example, an average of 3.2 partons are present at the end of the parton shower

\footnotetext{
${ }^{3}$ These values differ from the default ones for Herwig55, which also result from a fit to OPAL data, in that we now implement a new model feature which allows bottom baryons to be produced while maintaining a good description of the global event characteristics.
} 
in Cojets, compared to 9.1 in Jetset and 6.6 in Herwig. Thus it is unlikely that a gluon jet in a three-jet event from Cojets will evolve to such a degree, through additional gluon radiation, that its energy and angular structure will differ much from those of a quark jet: larger parton level differences are present for Jetset and Herwig. Gluons hadronize in a different manner from quarks in Cojets623, however, and so - as for Jetset and Herwig - differences are expected for this model between the hadron jets initiated by quarks and gluons. In contrast, quark and gluon jets hadronize in the same manner in Cojets612 and little difference is expected in this case between the properties of the two jet types. Comparison of the predictions of Cojets612 to the results of the other models thus provides a convenient means to illustrate the sensitivity of our analysis to differences between quark and gluon jet structure.

To evaluate the quark and gluon jet purities, we associate each simulated hadron jet with an underlying quark or gluon jet. To perform this association, we employ the following method. Monte Carlo events with detector simulation which pass the selection criteria given in sections 2 and 3 are examined at the parton level. The two hadron jets closest in angle to the directions of the primary quark and antiquark which have evolved from the $Z^{0}$ decay are considered to be the quark jets; the remaining jet is identified as the gluon jet. Note that we do not, in general, distinguish between a quark and an antiquark in this analysis.

The quark and gluon jet purities predicted for the normal-mixture and tagged samples are listed in table 4 . The values in this table state the quark jet content, $q$, in percent, for each of the jets; the gluon jet content, $g$, is given by $g=(100-q) \%$. Thus the Jetset-Peterson model predicts a purity of $g=80.0 \%$ for the gluon-tagged jets: the other models predict values which agree to better than $4 \%$ with the JetsetPeterson one. For the normal-mixture data, the lower energy jets are predicted to be about $51 \%$ quark and $49 \%$ gluon jets and the models agree to within $1 \%$, which is not surprising since in this case the purity values are mostly related to the event configuration and to the gluon bremsstrahlung spectrum, which is well known. We also include in table 4 the tagged event rate predicted by the models, defined by the ratio of the number of events in the tagged sample to that in the normal-mixture one, expressed in percent. The measured value of this quantity is $5.2 \pm 0.2 \%$. Thus Jetset predicts a rate which is slightly higher than that observed in the data, while the Herwig, Cojets and Jetset-Peterson values are somewhat lower. The generally lower rates observed in the Monte Carlo samples may imply that the background level is somewhat underestimated by the detector simulation program.

Since there is no unique prescription for determining whether a hadron jet is initiated by a quark or by a gluon, we tried a second method to make this association. For Monte Carlo events which satisfy the criteria of sections 2 and 3, three jets are reconstructed at the parton level, using the $k_{\perp}$ jet finder with a $y_{\text {cut }}$ value of 0.02 . About $6 \%$ of the events do not yield three jets with this $y_{\text {cut }}$ value but instead fall just outside this class. For the $6 \%$ of the events, an appropriate $y_{\text {cut }}$ value is chosen such that three parton level jets are always reconstructed. Each of the three 
parton jets is assigned to one of the hadron jets by finding the combination which minimizes the sum of the angular differences between them. The jets which contain the primary quark and antiquark, after their evolution has terminated, are taken to be the quark jets, while the remaining jet is assumed to be the gluon jet. The values for quark and gluon jet purity obtained from this second method agree with those obtained from the first to better than $1 \%$.

From table 4, we estimate the gluon-tagged jets to have a purity of $80.0 \pm 1.3 \pm$ $4.4 \%$, with a quark jet background of $20.0 \pm 1.3 \pm 4.4 \%$, where the first error is statistical and the second is systematic. For these estimates, we use the JetsetPeterson values because this model is known to provide a good description of the bottom hadron energy measurements. We consider three sources of systematic error: (1) the uncertainty in the mechanism of bottom hadron production and decay, (2) imperfections in the event reconstruction and detector simulation, and (3) the ambiguity of defining whether a jet arises from a quark or a gluon. The first uncertainty $(3.5 \%)$ is assessed by taking half of the maximum difference between the model predictions in table 4 . Since the generators are very different in their assumptions about bottom quark hadronization, we feel this yields a reasonable estimate of the uncertainty. For example, Jetset and Herwig present opposite extremes for the energy spectrum of bottom hadrons, as mentioned above. Jetset, Herwig and Cojets implement different models of hadronization. Cojets contains different tables, compared to Jetset and Herwig, to describe bottom hadron decays. Furthermore, the Jetset and Herwig samples were generated using a value of $c \tau=0.039 \mathrm{~cm}$ for the mean bottom hadron lifetime; for Cojets and Jetset-Peterson we used a larger value of $c \tau=0.042 \mathrm{~cm}^{4}$ The second source of systematic uncertainty, due to imperfect event reconstruction and detector simulation, is assessed by increasing the impact parameter resolution of tracks in the detector simulation program, using the JetsetPeterson model, so that its tagged event rate is $5.4 \%$ and therefore one standard deviation larger than is observed in the data. The difference between the purity values obtained using the modified and unmodified programs $(2.5 \%)$ is taken to be this uncertainty. The third source of systematic uncertainty, due to the ambiguity of defining a quark or a gluon jet $(0.7 \%)$, is given by the difference in the purity values obtained using the two methods to associate a hadron jet with a parton type, discussed above. The three sources of systematic error are added in quadrature to define the total systematic error.

Analogously, we estimate the composition of the lower energy jets in the normalmixture sample to be $48.5 \pm 0.2 \pm 0.8 \%$ gluon and $51.5 \pm 0.2 \pm 0.8 \%$ quark jets, where the systematic error is defined by the two sources (1) and (3) of systematic uncertainty listed above for the tagged data. We do not include the systematic error labelled (2) for this second case, since the impact parameter resolution is not relevant for the normal-mixture selection.

\footnotetext{
${ }^{4}$ These two values of $c \tau$ correspond to those published in 1990 [27] and 1992 [28], respectively, in the Review of Particle Properties.
} 


\subsection{Tests of the jet purities using data}

By requiring that a secondary vertex be present in one of the lower energy jets, we obtain a tagged data sample which contains an enhanced bottom quark component compared to the normal-mixture sample. A well known signal for the presence of bottom quarks is high energy leptons from semi-leptonic decay, which appear with large values of transverse momentum, $p_{T}$, with respect to the jet axis. Therefore a means to ascertain the relative fraction of bottom quarks in the tagged and normalmixture samples is to measure the ratio of the rates at which high momentum, high $p_{T}$ leptons appear in the highest energy jets of the two data sets. Since the purity value of the gluon-tagged jets is strongly correlated with the bottom quark fraction in the tagged sample, as discussed in section 3, this measurement provides a means to substantiate the purity estimates given in section 4.1.

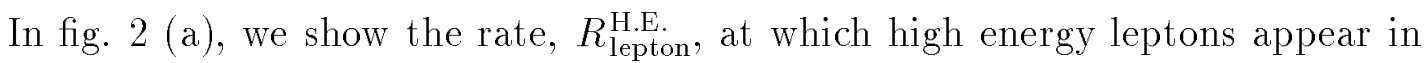
the highest energy jets of the tagged events, divided by the same quantity from the normal-mixture ones; $R_{\text {lepton }}^{\text {H.E. }}$ equals the number of electrons and muons with momentum values larger than $4 \mathrm{GeV} / c$ normalized by the number of events in the sample. Muons are identified in multihadronic events by associating tracks from the central detector with independently reconstructed track segments from the muon chambers. Electrons are identified by associating the central detector tracks with electromagnetic calorimeter clusters, as is discussed further in [29]. In the present case, electrons are accepted in the polar angle range $|\cos \theta|<0.7$, muons in the range $|\cos \theta|<0.9$ and the lepton is required to be within $30^{\circ}$ of the jet axis to which it is assigned. The data in fig. 2 (a) are displayed versus differential bins of $p_{T}$. The rightmost bin in fig. 2 (a) includes all $p_{T}$ values larger than $1.2 \mathrm{GeV} / c$.

For values of $p_{T}$ below about $1.0 \mathrm{GeV} / c$, there are substantial contributions to the lepton distribution from charm quark decays, bottom quark decays and background. The background is principally due to hadrons which are misidentified as leptons and leptons from the decay of light quark hadrons. For larger values of $p_{T}$, the contributions from charm quarks and background are expected to be small and the leptons are expected to arise primarily from bottom quark decays. For $p_{T}$ larger than $1.2 \mathrm{GeV} / c$, the ratio between the tagged and normal-mixture data in fig. 2 (a) is seen to have a value of about 2.5, which therefore sets a lower limit on the relative bottom quark population in the two data sets: only a lower limit is set, since there is still some background present, even for this bin. From the Jetset-Peterson model, we obtain a value of 3.0 for the ratio of the fraction of bottom quark jets in the highest energy jets of the two data sets: the other models yield similar results. Thus the observed lepton rates in the highest energy jets suggest that these jets contain a fraction of bottom quarks which is more than twice as large in the tagged sample as it is in the normal-mixture one, which accords well with the prediction from the Monte Carlo. The results from the Jetset-Peterson model, obtained by analyzing the Monte Carlo events in the same manner as the data, are shown by the hatched band in fig. 2 (a). The width of the band shows the statistical uncertainty, due to 
the finite Monte Carlo sample size. The Monte Carlo is seen to agree well with the measurements.

A similar but independent consistency test of the quark and gluon jet purity values is obtained by comparing the relative rate at which secondary vertices appear in the highest energy jets of the tagged and normal-mixture data. In fig. 2 (b), we show the ratio of the observed rates for these secondary vertices, displayed in

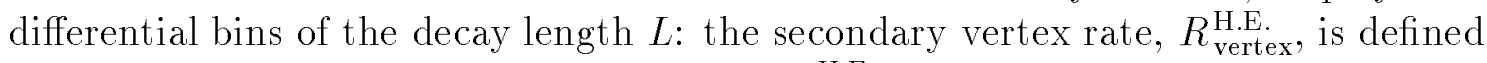
in an analogous manner to the lepton rate, $R_{\text {lepton }}^{\mathrm{H} \text {.E. }}$ discussed above. The rightmost bin in fig. 2 (b) includes all decay length values larger than $0.45 \mathrm{~cm}$. For values of $L$ below about $0.10 \mathrm{~cm}$, the distribution is dominated by background from light quark jets and the observed rates do not differ much between the two data sets. For values of $L$ above about $0.15 \mathrm{~cm}$, however, the secondary vertex rate is seen to be about three times larger in the tagged sample than it is in the normal-mixture one. The corresponding result from the Jetset-Peterson model is shown by the hatched band in fig. 2 (b). The Monte Carlo result agrees well with the data, again demonstrating that the enhancement factor for heavy quarks is well simulated. This provides a further consistency test - from the data - for the Monte Carlo purity values given in section 4.1 .

\section{$5 \quad$ Direct observation of quark-gluon jet differences}

We next compare the two lower energy jets in the normal-mixture sample to the gluon-tagged jets. Since these first jets are composed of about $49 \%$ gluon and $51 \%$ quark jets, as discussed in the section 4.1, while the corresponding proportions for the gluon-tagged jets are about $80 \%$ and $20 \%$, this comparison yields a direct and model independent test for differences between quark and gluon jets. The main assumption is that the gluon jets have the same properties in the two data sets, which we believe to be reasonable since these jets are selected using the same criteria in the two cases. ${ }^{5}$ Also, it is believed from QCD that a hard, acollinear gluon jet should be no different in a bottom quark event than in a light flavor one [30]. We also assume that the $20 \%$ background from quark jets, which is present in the gluontagged data, has the same properties as the quark jets in the normal-mixture sample. This is expected since most of the background events arise when a secondary vertex is reconstructed in the gluon jet, thus misidentifying it as a quark jet. In this case, there is no reason that one quark flavor should be favored over another, since the gluon jet is expected to have the same properties irrespective of the event flavor, as noted above. The second source of background is from the $3 \%$ of the events in which the gluon jet has the highest energy, recoiling against two quark jets in the opposite hemisphere. In this case, the vertex tagging preferentially selects heavy quark events compared to light quark ones; however, we find the overall background rate from

\footnotetext{
${ }^{5}$ We reemphasize that the jet which contains the identified secondary vertex is not included in our analysis.
} 
this source to be small and so make no correction for it (it contributes about $10 \%$ of the total background). We have also tested our assumptions using the Monte Carlo and find that there is essentially no difference between the properties of gluon jets selected using the secondary vertex criteria, compared to those selected from a normal-mixture sample in which the gluon jets are identified using the Monte Carlo information and mixed to include a $20 \%$ background from quark jets with normal properties. This illustrates that there is no important bias introduced into the jet properties by our analysis procedure. We note furthermore that quark mass effects, such as are discussed in section 3, do not alter the validity of our assumptions since they are not relevant for the gluon jets and since they affect the $20 \%$ quark jet background in the gluon-tagged sample in the same manner as they affect the quark jets in the normal-mixture events.

In the following, the distributions obtained separately for the two lower energy jets in the normal-mixture sample are averaged: we refer to this average as the normal-mixture jets. The normal-mixture jet sample therefore contains 45,274 entries and has small statistical errors compared to the 1175 jets of the gluon-tagged sample.

\subsection{Particle energy spectrum}

Figure 3 (a) shows the mean energy of the particles, $\langle E\rangle$, versus the azimuthal angle $\psi$ in the three-jet event plane. This distribution is constructed by weighting each particle with its energy before entering it at its position $\psi$, where $\psi$ is the angle in the event plane between the highest energy jet axis and the particle. The direction of the highest energy jet is obtained from the jet finder. Each bin is normalized by the number of particles in the bin after all events have been included. The histogram in fig. 3 (a) shows $\langle E\rangle$ versus $\psi$ for the normal-mixture jets, starting at the highest energy jet $\left(\psi \equiv 0^{\circ}\right)$, then proceeding through the lower energy jet $\left(\psi \approx 150^{\circ}\right)$ to stop halfway around the event plane $\left(\psi=180^{\circ}\right)$. The points with errors show $\langle E\rangle$ versus $\psi$ for the gluon jet side of the tagged sample, with their statistical uncertainties.

We define a "jet peak" from $120^{\circ}<\psi<180^{\circ}$ and a "jet core" from $135^{\circ}<\psi<$ $165^{\circ}$. These regions are delineated by the dashed and dotted lines, respectively, in fig. 3 (a). This definition of the jet peak differs slightly from that used in [6] because we now wish this interval to be symmetric around $\psi=150^{\circ}$. A small shift is visible in fig. 3 (a) between the peak positions of the gluon-tagged and normal-mixture jets. This is due to the finite bin size of $\pm 10^{\circ}$ used to define symmetric events and to the nature of the bremsstrahlung spectrum, which tends to shift the gluon jets to smaller values of energy and $\psi$. Since the energies of the normal-mixture and gluon-tagged jets are the same to better than $2 \%$, this small shift does not affect our direct comparisons in a significant manner.

It is seen from fig. 3 (a) that the mean particle energy value of the gluon-tagged 
jets is smaller than it is for the normal-mixture jets in the jet core, while no such difference is seen elsewhere in the jet peak. This is consistent with what was first observed in [6]. The $\chi^{2}$ value between the two distributions is 38.5 (12 bins) for the jet peak and 35.4 ( 6 bins) for the jet core. We therefore observe a clear signal for quark-gluon jet differences: the overall particle energy spectrum of gluon jets is softer than it is for quark jets, as expected from QCD.

Besides the difference in $\langle E\rangle$ for $\psi \sim 150^{\circ}$, there is a visible difference between the two data sets in the region of the highest energy jets, $0^{\circ}<\psi<30^{\circ}$. The points with errors in this region represent an enhanced sample of bottom quark jets, whereas the histogram corresponds approximately to a normal flavor mixture of quark jets at the $\mathrm{Z}^{0}$ peak. ${ }^{6}$ However, bottom jets are known to yield a larger mean particle multiplicity than jets from lighter quark types [31]. The value of $\langle E\rangle$ in fig. 3 (a) for $\psi<30^{\circ}$ is thus smaller for the bottom-enhanced quark jets than it is for the normal-mixture ones - as required by conservation of energy - since the jet energies are the same in the two cases. This difference for the highest energy jets does not affect our study since we only assume the gluon jet properties and their background to be the same in the two data sets, as mentioned in the introduction to this section. Consider, for example, the distributions shown in fig. 3 (b), obtained using the Jetset-Peterson model including detector simulation. The points with errors show the gluon jet side of events in which the Monte Carlo is treated like the tagged data sample. The histogram shows normal-mixture events for which the quark and gluon jets are identified using the Monte Carlo information, and for which the gluon and quark jet sides are selected randomly event-by-event to correspond to the $80 \%$ and $20 \%$ gluon and quark jet composition of the tagged sample. In the region of the highest energy jet, the same differences are visible as are seen for the data in fig. 3 (a). The gluon jet peak at $\psi \approx 150^{\circ}$ is virtually identical for the two cases, however, demonstrating the absence of an important bias in our analysis. We find similar results for the other distributions which we study.

The softness of the gluon jet particle energy spectrum with respect to that of the quark jet is further illustrated in fig. 4 (a), which shows the inclusive scaled energy distribution of particles, $\left(1 / n_{\text {total }}\right) \mathrm{d} n_{\text {total }} / \mathrm{d} x_{E}$, for the normal-mixture (histogram) and gluon-tagged (points with errors) jets. The particle assignments to a jet are taken from the jet finder; $x_{E}=E / E_{\text {vis. }}^{\text {jet }}$ with $E$ the particle energy and $E_{\text {vis. }}^{\text {jet }}$ the visible jet energy. The ratio of the gluon-tagged to the normal-mixture measurements is shown in 4 (b). The $\chi^{2}$ value between the two curves is 90.5 (9 bins).

\footnotetext{
${ }^{6}$ From the Monte Carlo, we estimate the flavor composition of the tagged sample to be $61 \%$ bottom quarks, $15 \%$ charm quarks and $24 \%$ strange, up or down quarks; the normal-mixture sample is predicted to contain about $20 \%$ bottom quarks, $22 \%$ strange and down quarks and $18 \%$ charm and up quarks.
} 


\subsection{Particle multiplicity}

Since the quark and gluon jets in our study have the same energy, while the particle energy spectrum of gluon jets is observed to be softer, the gluon jets can be expected to exhibit a larger mean multiplicity. Figure 5 (a) shows the multiplicity of tracks and clusters assigned to the gluon-tagged and normal-mixture jets by the jet finder, $n_{\text {total }}$. The mean values are $13.76 \pm 0.14$ and $12.73 \pm 0.02$, respectively; the ratio between them is $1.081 \pm 0.011$, which exceeds unity by 7.4 standard deviations (s.d.). The distributions for charged tracks alone, $n_{\mathrm{ch}}$, are shown in fig. 5 (b), for which the corresponding values are $8.62 \pm 0.10$ and $7.90 \pm 0.02$, giving the ratio $1.092 \pm 0.013$ which is 7.1 s.d. larger than unity. Thus the gluon jets do indeed yield a larger multiplicity than the quark jets.

Since we rely on the jet finder to determine the particle assignments to a jet, our results can depend on the choice of the jet algorithm. For example, the $k_{\perp}$ algorithm occasionally assigns soft particles to a jet which lies in the opposite hemisphere. This feature especially affects the multiplicity distribution, compared to the other distributions which we study, since it is dominated by soft particles. Following the suggestions of [32], we therefore reassign the particles in an event to the nearest jet axis - originally found using the $k_{\perp}$ scheme - and then recompute the jet axes to be the vector sum of the constituent particle momenta. This procedure is iterated until the particle assignments are stable. On average, 1.4 particles per event are reassigned to a different jet axis; the highest energy jet changes in direction by a mean of $0.9^{\circ}$ and the two lower energy jets by $1.7^{\circ}$. The estimated purity levels of the quark and gluon jets remain essentially unchanged, as determined by applying the reassignment procedure at both the parton and hadron levels in the Monte Carlo and then re-performing the quark and gluon jet association discussed in section 4.1. For the modified jet definition, we obtain mean values for particle multiplicity of $13.46 \pm 0.12$ and $12.72 \pm 0.02$ for the gluon-tagged and normal-mixture jets, using the charged tracks and clusters. For charged tracks alone, the corresponding results are $8.42 \pm 0.09$ and $7.89 \pm 0.02$. The two cases yield ratios of $1.058 \pm 0.010$ and $1.068 \pm 0.012$, respectively, which differ from unity by 5.8 and 5.7 s.d. We thus observe a smaller difference between quark and gluon jet multiplicities using the modified assignments: the difference between the mean multiplicity values remains significant, however.

In our previous study of quark and gluon jet differences [6], we did not use the jet finder assignments to associate particles with a jet, but instead divided each event into hemispheres using the plane which was perpendicular to the three-jet event plane and which contained the highest energy jet axis. This resulted in distributions analogous to fig. 3 (a). The mean multiplicity values of the gluon-tagged and normal-mixture jets were determined by integrating the corresponding multiplicity distributions over the jet peak regions, which were defined in a similar manner to that indicated by the dashed line in fig. 3 (a). Therefore particles were always associated with a jet in the same hemisphere. In this sense, our previous method is 
similar to the alternative jet definition discussed in the previous paragraph, based on the nearest angle between a jet and a particle. Thus it is appropriate to compare the results obtained with this alternative jet definition to our previous results. In our earlier study, we found a ratio of $1.03 \pm 0.03$ (stat.) and $1.02 \pm 0.04$ (stat.) between the mean multiplicities of gluon and quark jets, for the total and charged multiplicities, respectively. These data were not corrected for quark and gluon jet misidentification and so are comparable to those presented in this section. ${ }^{7}$ Our previous result therefore agrees with the result presented above which uses the reassigned particles, to within about one standard deviation of the statistical error. If we apply our previous method of determining a jet's multiplicity to the data samples discussed in the present study, we obtain ratios of $1.051 \pm 0.009$ and $1.056 \pm 0.015$ between the gluon-tagged and normal-mixture jets, for all particles and for charged particles alone, respectively. These last values also agree well with our previous results and with those based on the alternative jet definition discussed above.

\section{$5.3 \quad$ Jet widths}

A common measure of the broadness of a jet is the fraction of its visible energy, $f_{E}(\theta)$, which lies within a cone of half-angle $\theta$ around the jet axis [33]:

$$
f_{E}(\theta)=\frac{\int_{\theta^{\prime}=0^{\circ}}^{\theta^{\prime}=\theta}\left(\mathrm{d} E_{\text {vis. }}^{\text {jet }} / \mathrm{d} \theta^{\prime}\right) d \theta^{\prime}}{E_{\text {vis. }}^{\text {jet }}}
$$

In fig. 6, we show the distribution of the fraction of the visible energy of a jet contained within a cone of half angle $\theta=10^{\circ}$ around the jet axes, for the normalmixture and gluon-tagged jets. The normal-mixture jets are observed to be more peaked toward large values of $f_{E}(\theta)$ than are the gluon-tagged ones. Therefore gluon jets have a smaller fraction of their energy close to the jet axis than quark jets and in this sense are broader. Again, this is in qualitative agreement with the expectations of QCD. The $\chi^{2}$ value between the normal-mixture and gluon-tagged distributions is 130 (14 bins).

Figure 7 (a) shows the mean value of the fractional jet energy, $\left\langle f_{E}(\theta)\right\rangle$, versus the cone half angle $\theta$, for $0^{\circ}<\theta<45^{\circ}$. The fractional difference between the two curves, defined by

$$
d_{E}(\theta) \equiv \frac{\left\langle f_{E}(\theta)\right\rangle_{\text {n.mix }}-\left\langle f_{E}(\theta)\right\rangle_{\text {g.tag }}}{\left\langle f_{E}(\theta)\right\rangle_{\text {g.tag }}}
$$

is shown in fig. 7 (b), where the subscripts in eq. (3) refer to the normal-mixture (n.mix) and gluon-tagged (g.tag) data. The errors between the different bins of fig. 7 are correlated since each bin contains the integral over smaller $\theta$ values. In

\footnotetext{
${ }^{7}$ In our previous study, the estimated purity values of the gluon and quark jets were about $80 \%$ for both jet types: since the ratios observed are close to unity, the difference in estimated purities with respect to the present study does not much affect their values.
} 
figs. 8 (a) and (b) we show the corresponding differential distributions, $f_{E}^{\text {diff. }}=$ $\left(1 / E_{\text {vis. }}^{\text {jet }}\right) \mathrm{d} E_{\text {vis. }}^{\text {jet }} / \mathrm{d} \theta$ and $d_{E}^{\text {diff. }}$, versus $\theta$. The quark jets are seen to have a larger energy density for $\theta<10^{\circ}$ while the energy density of the gluon jets is larger for $\theta>10^{\circ}$.

Another common measure of the width of a jet is the total particle momentum normal to the jet axis. Distributions in total transverse particle momentum can be resolved into orthogonal components in and out of the three-jet event plane, $p_{\mathrm{T}}^{\mathrm{in}}$ and $p_{\mathrm{T}}^{\text {out }}$, and they thus yield additional, detailed information about jet structure. Constructing these distributions by using the particle assignments to jets from the $k_{\perp}$ jet algorithm, we find mean values for $p_{\mathrm{T}}^{\text {in }}$ of $3.58 \pm 0.06 \mathrm{GeV} / c$ and $3.26 \pm$ $0.01 \mathrm{GeV} / c$ and for $p_{\mathrm{T}}^{\text {out }}$ of $3.59 \pm 0.05 \mathrm{GeV} / c$ and $3.25 \pm 0.01 \mathrm{GeV} / c$, for the gluon-tagged and normal-mixture data, respectively: the ratios between them are $1.098 \pm 0.018$ and $1.107 \pm 0.017$ for the two directions. The broadness of gluon jets relative to quark jets is therefore present in both components and has about the same relative magnitude in both cases. This is consistent with our earlier results [6]. If the mean values of particle transverse momentum are considered, $p_{\mathrm{T}}^{\mathrm{in}} / n_{\text {total }}$ and $p_{\mathrm{T}}^{\text {out }} / n_{\text {total }}$, we find means of $0.260 \pm 0.004 \mathrm{GeV} / c$ and $0.254 \pm 0.001 \mathrm{GeV} / c$ for the component in the event plane and $0.262 \pm 0.003 \mathrm{GeV} / c$ and $0.254 \pm 0.001 \mathrm{GeV} / c$ for the component out of the event plane, for the gluon-tagged and normal-mixture jets: the ratios between the gluon-tagged and normal-mixture data are therefore $1.024 \pm$ 0.014 and $1.035 \pm 0.013$, respectively. The smaller difference of about $3 \%$ which is observed between the two jet types when the mean transverse momentum values are considered, relative to the $11 \%$ difference noted above for the total transverse momentum values, demonstrates that most of the difference for this second case is due to the different mean multiplicities of the two jet types and not to the difference in their energy spectra.

\section{Corrected distributions}

We next correct the distributions presented in section 5 for the residual quark-gluon jet misidentification, to obtain distributions valid for pure quark and pure gluon jet states. If $D_{\text {g.tag }}\left(z_{i}\right)$ and $D_{\text {n.mix }}\left(z_{i}\right)$ represent the gluon-tagged and normal-mixture data, with $z_{i}$ the content of bin $i$, we may write

$$
\begin{aligned}
& D_{\text {g.tag }}\left(z_{i}\right)=\left(1-q_{\text {g.tag }}\right) \cdot G\left(z_{i}\right)+q_{\text {g.tag }} \cdot Q\left(z_{i}\right) ; \\
& D_{\text {n.mix }}\left(z_{i}\right)=\left(1-q_{\text {n.mix }}\right) \cdot G\left(z_{i}\right)+q_{\text {n.mix }} \cdot Q\left(z_{i}\right) ;
\end{aligned}
$$

where $G\left(z_{i}\right)$ and $Q\left(z_{i}\right)$ are the distributions for pure gluon and pure quark jets, respectively. The values of the coefficients $q_{\text {g.tag }}$ and $q_{\text {n.mix }}$ were presented in section 4:

$$
q_{\text {g.tag }}=0.200 ; q_{\text {n.mix }}=0.515 .
$$

Knowledge of these coefficients permits relations (4) to be inverted for $G\left(z_{i}\right)$ and $Q\left(z_{i}\right)$. Note that these coefficients are the only Monte Carlo information used to 
correct the data. We do not correct them for detector acceptance and resolution in order to keep the Monte Carlo dependence of the results as small as possible.

In figs. 9-14, we show the results of figs. 3-8 after correcting them in this manner. The bin-to-bin statistical fluctuations of the quark jet state, $Q\left(z_{i}\right)$, are about the same size and are anti-correlated with those of the gluon jet state, $G\left(z_{i}\right)$. The statistical significance of the differences we observe between quark and gluon jets is therefore more readily seen from figs. 3-8, without corrections, while the size of those differences is shown in figs. 9-14. Included in figs. 9-14 are the predictions of the QCD Monte Carlo programs discussed in section 4.1. The Monte Carlo samples include full detector simulation and the same selection criteria as the data, except that quark and gluon jets have been identified using the Monte Carlo information. The simulated events therefore approximately represent a normal mixture of quark jet flavors at the $Z^{0}$ peak. The Jetset and Jetset-Peterson predictions are very similar and so we only include the Jetset-Peterson results in figs. 9-14 and in the discussion below.

The corrected mean particle energy distribution, $\langle E\rangle$ versus $\psi$, is shown for the quark jets in fig. 9 (a); the corresponding distribution for the gluon jets is shown in fig. 9 (b). In the region of the jet core, around $\psi \sim 150^{\circ}$, the smaller mean particle energy value of the gluon jets, relative to the quark jets, is clear. Jetset-Peterson, Herwig and Cojets623 provide satisfactory descriptions of the data, although the quark jet distribution is better described by the first two models than by the last. Cojets612 does not contain significant quark-gluon jet differences, as discussed in section 4.1. It does not describe the data, as is especially clear for the gluon jet. This demonstrates the sensitivity of this distribution to differences between quark and gluon jet structure. The differences between the corrected data and models seen for the highest energy jets, corresponding to $0^{\circ}<\psi<30^{\circ}$ in fig. 9 , are due to the enhanced bottom quark population which is present for these jets in the tagged sample, relative to the normal-mixture sample, as discussed in section 5.1: the correction equations (4) are not valid in this region. These differences for the highest energy jets do not influence our study, as explained in section 5.1. Figure 10 (a) shows the inclusive particle energy spectrum, $\left(1 / n_{\text {total }}\right) \mathrm{d} n_{\text {total }} / \mathrm{d} x_{E}$, for the corrected data; the ratio between the gluon and quark jet curves is shown in fig. 10 (b). The errors in fig. 10 (b) take into account the correlations between $G\left(z_{i}\right)$ and $Q\left(z_{i}\right)$. Particles with large values of $x_{E}\left(x_{E}>0.45\right)$ are seen to occur in gluon jets at only about one quarter the rate that they occur in quark jets. Again, the data are adequately described by the models which include significant quark-gluon jet differences, but not by Cojets612.

Figure 11 displays the corrected particle multiplicity distributions of the jets, using the $k_{\perp}$ jet finder assignments. The mean values for $G\left(z_{i}\right)$ and $Q\left(z_{i}\right)$, including charged tracks and unassociated clusters (fig. 11 (a) and (b)), are $\left\langle n_{\text {total }}\right\rangle_{\text {gluon }}=$ $14.41 \pm 0.13$ and $\left\langle n_{\text {total }}\right\rangle_{\text {quark }}=11.37 \pm 0.13$, respectively. For charged tracks alone (fig. $11(\mathrm{c})$ and $(\mathrm{d})$ ), we obtain $\left\langle n_{\text {ch. }}\right\rangle_{\text {gluon }}=9.10 \pm 0.10$ and $\left\langle n_{\text {ch. }}\right\rangle_{\text {quark }}=6.86 \pm 0.09$. 
These lead to ratios of

$$
\begin{aligned}
& \left.\frac{\left\langle n_{\text {total }}\right\rangle_{\text {gluon }}}{\left\langle n_{\text {total }}\right\rangle_{\text {quark }}}=1.267 \pm 0.043 \text { (stat. }\right) \pm 0.055 \text { (syst.) } ; \\
& \left.\frac{\left\langle n_{\text {ch. }}\right\rangle_{\text {gluon }}}{\left\langle n_{\text {ch. }}\right\rangle_{\text {quark }}}=1.326 \pm 0.054 \text { (stat. }\right) \pm 0.073 \text { (syst.) },
\end{aligned}
$$

where the statistical errors take into account the correlations between the numerator and denominator. The systematic error has two sources: (1) the uncertainty in the values of the correction coefficients, discussed in section 4, and (2) the maximum variation in the result which is observed when we choose a decay length interval of 0.10 to $0.50 \mathrm{~cm}$, or of 0.15 to $0.30 \mathrm{~cm}$, to tag quark jets, instead of the interval of 0.15 to $0.50 \mathrm{~cm}$ used for the rest of our analysis. The two sources of systematic uncertainty contribute values of 0.042 and 0.035 , respectively, to the systematic error of the total multiplicity ratio; the corresponding values for charged tracks alone are 0.060 and 0.042 . Thus the gluon jets yield a particle multiplicity which is about 30\% larger than the quark jets. Once again, Jetset-Peterson, Herwig and Cojets623 provide a satisfactory description of the data, although the quark jet is better described by Jetset-Peterson and Herwig than it is by Cojets. In contrast, Cojets612 does not describe the measurements, especially not those of the gluon jet. The ratio of mean multiplicity between the gluon and quark jets is found to be $1.26,1.21,1.16$ and 0.95 for the four models, respectively, with an error of 0.01 in all cases due to limited Monte Carlo statistics. The models give the same results for charged tracks alone as they do when the unassociated clusters are included. The Monte Carlo results for the multiplicity ratios are, in addition, found to be the same at the event generator level as they are when detector simulation and selection cuts are included, to within about 0.02: thus effects related to detector acceptance and resolution provide only a small correction to these ratios. If the alternative method to associate particles and jets is employed, based on reassigning particles to the jet to which they are nearest in angle, as discussed in section 5.2, the corrected multiplicity ratios (6) become $1.19 \pm 0.04$ (stat.) for the total multiplicity and $1.20 \pm 0.04$ (stat.) for the charged multiplicity, which are smaller than the values obtained using the $k_{\perp}$ jet finder assignments. The numerical result for the ratio values is therefore sensitive to the choice of the jet algorithm.

In our earlier publication [6], based on lepton tagging of quark jets and a much smaller data sample, the data were not corrected for quark and gluon jet misidentification and so cannot be directly compared to those presented in this section. Also, the jet definitions used in our previous and present studies are not the same. Our previous results are consistent with the present ones when compared under similar conditions, as discussed in section 5.2.

The corrected jet cone energy fraction distribution, $f_{E}(\theta)$, is shown in fig. 12 for $\theta=10^{\circ}$. The quark jet distribution peaks toward large values of $f_{E}(\theta)$ while the gluon jet distribution peaks toward small values: thus the jet energy is more tightly collimated around the jet axis for quark jets than it is for gluon jets. Again 
the models - with the exception of Cojets612 - provide a satisfactory description of the measurements, although Cojets623 does not describe the quark jets as well as Jetset-Peterson and Herwig. Figures 13 (a) and 14 (a) show the corrected mean fractional jet energy distribution in its integrated and differential form, $\left\langle f_{E}(\theta)\right\rangle$ and $\left(1 / E_{\text {vis. }}^{\text {jet }}\right) \mathrm{d} E_{\text {vis. }}^{\text {jet }} / \mathrm{d} \theta$, versus $\theta$. The quark jets are observed to have an average of about $31 \%$ of their energy enclosed within a cone of half angle $5^{\circ}$ around the jet axis, compared to only $14 \%$ for gluon jets. The corresponding fractional differences, $d_{E}(\theta)$ and $d_{E}^{\text {diff. }}$, are shown in figs. 13 (b) and $14(\mathrm{~b})$; the errors shown take into account the correlations between $G\left(z_{i}\right)$ and $Q\left(z_{i}\right)$. It is seen from these last figures that there is a larger difference between quark and gluon jet widths in the data than in the models. This was also observed in [6], using a different technique to measure the jet widths: the general shape of the measured distribution is well represented by Jetset-Peterson, Herwig and Cojets623, however. In contrast, Cojets612 yields an essentially flat curve in figs. 13 (b) and 14 (b) and is in marked disagreement with the data, as expected.

We have tested the stability of our results to the event definition, by repeating the analysis using a narrower angular range to define the symmetric three-jet topology. We required the angle between the highest energy jet and the two others to be in the range $150 \pm 5^{\circ}$, instead of the range $150 \pm 10^{\circ}$ used otherwise. With the more restrictive geometrical condition, the three-jet event sample was reduced in size by approximately a factor of four, but it yielded consistent results with those presented above. In particular, the ratio of the gluon to quark jet mean multiplicities remained the same to better than one standard deviation of the statistical error given above. We also tested the sensitivity of our results to the choice of the $y_{c u t}$ value used with the $k_{\perp}$ jet finder, by repeating the analysis using $y_{c u t}=0.04$ instead of $y_{c u t}=$ 0.02. Again, we found essentially identical results to those presented above and the multiplicity ratios remained within one standard deviation of the statistical error given in (6). As a last note, we point out that although the distributions presented here are not corrected for detector acceptance and resolution, these corrections are small for several of the quantities in our study. This is true for the multiplicity ratios of gluon to quark jets, as discussed above. Likewise, the jet width distributions, figs. 12-14, are found to have essentially negligible detector corrections.

\section{$7 \quad$ Summary and discussion}

In this paper, we have studied three-jet events from hadronic $Z^{0}$ decays in which the energies and environments of the two lowest energy jets are the same. This type of analysis was first introduced by our collaboration in two earlier publications [6, 8]. Here, we extend our previous ideas by using reconstructed secondary vertices in multi-jet events to perform the quark and gluon jet separation. Use of the symmetric events allows the properties of the quark and gluon jets to be compared in a simple and direct manner. We do not require Monte Carlo calculations in order to establish 
the experimental results. Monte Carlo models are used to estimate the quark and gluon jet misidentification level, to illustrate that the jet properties are not biased by the analysis technique and to demonstrate the sensitivity of the chosen distributions to differences between quark and gluon jet structure. The experimental results stand on their own, however, and are entirely model independent.

For the jets with energies of about $24 \mathrm{GeV}$ which we study, we observe significant differences between quark and gluon jet properties. The particle energy spectrum of gluon jets is softer, their multiplicity is larger, and their energy is less concentrated near the jet core compared to quark jets: thus, the gluon jets are broader. These features are in qualitative agreement with the expectations of perturbative QCD. The ratio of 1.27 which we observe between the gluon and quark jet mean multiplicities, after correcting for the residual quark and gluon jet misidentification, is considerably smaller than the naïve theoretical value of $9 / 4$ mentioned in the introduction. Recently, an analytic calculation of particle multiplicity in two- and three-jet events from $\mathrm{e}^{+} \mathrm{e}^{-}$annihilations has been presented [4], which demonstrates that higher orders and cancellations associated with coherent soft gluon emission substantially reduce the theoretical expectation for this ratio. However, this calculation is performed for a general class of events rather than for the geometrically symmetric ones which we study and so is not directly comparable to our measurement. The multiplicity result also depends on the jet definition: if we assign particles to the jet to which they are nearest in angle, rather than use the assignments from the jet finder, we find a smaller ratio of about 1.20 between the quark and gluon jet mean multiplicities. The result found here is entirely consistent with that obtained in our previous study, if a similar method of associating the soft particles to a jet is employed.

We also compared our results to model calculations which incorporate perturbative QCD. At the hadron level, Jetset and Herwig describe the quark and gluon jet measurements quite well. The description of the gluon jet by Cojets, version 6.23, is also good, but this model does not describe the quark jet as accurately as the other two models. It is possible that the Cojets description of the quark jet could be improved by a simple parameter adjustment. Cojets, version 6.12 , which does not contain significant quark-gluon jet differences, is in clear disagreement with the data, as expected.

The Monte Carlo models contain detailed simulations of QCD radiation and interference. It is therefore interesting to examine their predictions at a more fundamental level, to obtain insight into possible interpretations of our observations. At the parton level, using the quark and gluon four-momenta after termination of their evolution, Jetset and Herwig predict qualitative differences between quark and gluon jets which are similar to those which they yield at the hadron level. This suggests that the observed differences have a perturbative origin, at least in part. Using the same jet finder and symmetric three-jet condition as we apply at the hadron level, the parton level multiplicity ratios of gluon to quark jets are found to be 1.29 and 1.19 for Jetset and Herwig, respectively. For the mean multiplicity 
values at the parton level, we sum the number of partons associated with each jet after termination of the perturbative stage and then subtract unity so that the contribution of the initiating quark or gluon itself is not included: the multiplicity ratio which results corresponds more closely to the theoretical calculations [1] than if the initiating quark or gluon is included in the sums. These ratios are very similar to the corresponding ones found at the hadron level for the two models, which were given in section 6 . The differences observed between quark and gluon jets using the partons are not entirely the same, however, as those observed using the hadrons. For example, the differential jet cone energy distributions of quark and gluon jets cross each other at an angle of about $10^{\circ}$ at the hadron level, as seen from figs. 8 or 14. At the parton level, we observe that the two curves cross at a smaller angle of about $5^{\circ}$, for both Jetset and Herwig. This suggests that hadronization also plays an important role in explaining the quantitative features of the observed quark-gluon jet differences.

\section{Acknowledgements}

We thank Steve Ellis for interesting discussions. It is a pleasure to thank the SL Division for the efficient operation of the LEP accelerator and their continuing close cooperation with our experimental group. In addition to the support staff at our own institutions we are pleased to acknowledge the

Department of Energy, USA,

National Science Foundation, USA,

Texas National Research Laboratory Commission, USA,

Science and Engineering Research Council, UK,

Natural Sciences and Engineering Research Council, Canada,

Fussefeld Foundation,

Israeli Ministry of Energy,

Israeli Ministry of Science,

Minerva Gesellschaft,

Japanese Ministry of Education, Science and Culture (the Monbusho) and a grant under the Monbusho International Science Research Program,

German Israeli Bi-national Science Foundation (GIF),

Direction des Sciences de la Matière du Commissariat à l'Energie Atomique, France, Bundesministerium für Forschung und Technologie, FRG,

National Research Council of Canada, Canada,

A.P. Sloan Foundation and Junta Nacional de Investigação Científica e Tecnológica, Portugal. 


\section{References}

[1] S.J. Brodsky and J. Gunion, Phys. Rev. Lett. 37 (1976) 402;

K. Konishi, A. Ukawa and G. Veneziano, Phys. Lett. B78 (1978) 243.

[2] K.Shizuya and S.-H.H. Tye, Phys. Rev. Lett. 41 (1978) 787;

M.B. Einhorn and B.G. Weeks, Nucl. Phys. B146 (1978) 445;

H. P. Nilles and K. H. Streng, Phys. Rev. D23 (1981) 1944;

A.H. Mueller, Nucl. Phys. B241 (1984) 141;

J.B. Gaffney and A.H. Mueller, Nucl. Phys. B250 (1985) 109.

[3] Yu.L. Dokshitzer, V.A. Khoze and S.I. Troyan, in Perturbative Quantum Chromodynamics, ed. A. Mueller (World Scientific, Singapore) 1989;

Yu.L. Dokshitzer, V.A. Khoze, A.H. Mueller and S.I. Troyan, Basics of Perturbative QCD (Editions Frontières, Paris) 1991.

[4] S. Catani, B.R. Webber, Yu.L. Dokshitzer and F. Fiorani, CERN.TH.6419/92.

[5] JADE Collaboration, W. Bartel et al., Phys. Lett. 123B (1983) 460;

UA2 Collaboration, P. Bagnaia et al., Phys. Lett. 144B (1984) 291;

HRS Collaboration, M. Derrick et al., Phys. Lett. 165B (1985) 449;

MARK2 Collaboration, A. Petersen et al., Phys. Rev. Lett. 55 (1985) 1954;

UA1 Collaboration, G. Arnison et al., Nucl. Phys. B276 (1986) 253;

TASSO Collaboration, W. Braunschweig et al., Z. Phys. C45 (1989) 1;

AMY Collaboration, Y. K. Kim et al., Phys. Rev. Lett. 63 (1989) 1772.

[6] OPAL Collaboration, G. Alexander et al., Phys. Lett. B265 (1991) 462.

[7] D. Amati and G. Veneziano, Phys. Lett. B83 (1979) 87;

Y.I. Azimov, Yu.L. Dokshitzer, V.A. Khoze and S.I. Troyan, Z. Phys. C27 (1985) 65 .

[8] OPAL Collaboration, M.Z. Akrawy et al., Phys. Lett. B261 (1991) 334.

[9] OPAL Collaboration, K. Ahmet et al., Nucl. Instr. and Meth. A305 (1991) 275.

[10] P.P. Allport et al., "The OPAL Silicon Microvertex Detector", to be published in Nucl. Instr. and Meth.

[11] M. Arignon et al., Nucl. Instr. and Meth. A313 (1992) 103.

[12] D. Charlton et al., CERN-PPE/92-136, to be published in Nucl. Instr. and Meth.

[13] S. Catani et al., Phys. Lett. B269 (1991) 432;

N. Brown and W. J. Stirling, Z. Phys. C53 (1992) 629;

S. Bethke, Z. Kunszt, D. Soper and W.J. Stirling, Nucl. Phys. B370 (1992) 310. 
[14] J.D. Bjorken and S.J. Brodsky, Phys. Rev. D1 (1970) 1416;

SLAC-LBL Collaboration, G. Hanson et al., Phys. Rev. Lett. 35 (1975) 1609.

[15] L3 Collaboration, B. Adeva et al., Phys. Lett. B271 (1991) 461.

[16] OPAL Collaboration, P.D. Acton et al., Phys. Lett. B273 (1991) 355.

[17] J. Allison et al., Nucl. Instr. and Meth. A317 (1992) 47.

[18] T. Sjöstrand, Comp. Phys. Comm. 39 (1986) 347;

T. Sjöstrand and M. Bengtsson, Comp. Phys. Comm. 43 (1987) 367;

T. Sjöstrand, CERN-TH.6488/92.

[19] G. Marchesini, B.R. Webber et al., Comp. Phys. Comm. 67 (1992) 465.

[20] R. Odorico, Comp. Phys. Comm. 32 (1984) 139; Comp. Phys. Comm. 59 (1990) 527.

[21] OPAL Collaboration, M. Z. Akrawy et al., Z. Phys. C47 (1990) 505.

[22] P. Mazzanti and R. Odorico, Nucl. Phys. B370 (1992) 23; Bologna report DFUB 92/1 (1992).

[23] See, for example, T. Sjöstrand et al. in "Z Physics at LEP 1", ed. G. Altarelli, R. Kleiss and C. Verzegnassi, CERN-89-08, vol. 3, (Geneva, 1989).

[24] J.H. Kühn, P.M. Zerwas et al., in "Z Physics at LEP 1", ed. G. Altarelli, R. Kleiss and C. Verzegnassi, CERN-89-08, vol. 1, (Geneva, 1989).

[25] C. Peterson, D. Schlatter, I. Schmitt and P. Zerwas, Phys. Rev. D27 (1983) 105.

[26] ALEPH Collaboration, D. Decamp et al., Phys. Lett. B244 (1990) 551;

L3 Collaboration, B. Adeva et al., Phys. Lett. B261 (1991) 177.

[27] Particle Data Group, "Review of Particle Properties", Phys. Lett. B239, (1990) 1.

[28] Particle Data Group, "Review of Particle Properties", Phys. Rev. D45, part 2 (1992).

[29] OPAL Collaboration, P.D. Acton et al., Z. Phys. C55 (1992) 191.

[30] V. Khoze in Proc. Int. Symp. Lepton Photon Interactions, Stanford 1989, ed. M. Riordan (World Scientific, Singapore) 1990.

[31] MARK2 Collaboration, P. Rowson et al., Phys. Rev. Lett. 54 (1985) 2580.

[32] J. Pumplin, Michigan State University preprint MSUTH 92/04.

[33] See, for example, L.M. Jones, Phys. Rev. D39 (1989) 2550. 


\begin{tabular}{|c|c|c|c|}
\hline \multicolumn{4}{|c|}{ Normal-mixture selection } \\
\hline & Calculated energy & Visible energy & Angle with H.E. jet \\
\hline Highest energy jets & $42.45 \pm 0.01 \mathrm{GeV}$ & $33.70 \pm 0.05 \mathrm{GeV}$ & - \\
\hline Lower energy jets & $24.39 \pm 0.01 \mathrm{GeV}$ & $21.68 \pm 0.02 \mathrm{GeV}$ & $151.1 \pm 0.1^{\circ}$ \\
\hline \multicolumn{4}{|c|}{ Tagged selection } \\
\hline & Calculated energy & Visible energy & Angle with H.E. jet \\
\hline Highest energy jets & $42.43 \pm 0.03 \mathrm{GeV}$ & $33.68 \pm 0.21 \mathrm{GeV}$ & - \\
\hline Gluon-tagged jets & $23.96 \pm 0.09 \mathrm{GeV}$ & $21.58 \pm 0.14 \mathrm{GeV}$ & $150.4 \pm 0.2^{\circ}$ \\
\hline Jets with vertex & $24.85 \pm 0.09 \mathrm{GeV}$ & $21.42 \pm 0.15 \mathrm{GeV}$ & $151.6 \pm 0.2^{\circ}$ \\
\hline
\end{tabular}

Table 1: Mean calculated jet energies, mean visible energies and mean angles with respect to the highest energy (H.E.) jets, for the jets in the normal-mixture and tagged data samples.

\begin{tabular}{|c|c|c|c|c|}
\hline \multicolumn{5}{|c|}{ Herwig55 parameter values } \\
\hline \hline QCDLAM & VGCUT & VQCUT & CLMAX & CLPOW \\
\hline $0.18 \mathrm{GeV}$ & $0.10 \mathrm{GeV}$ & $0.48 \mathrm{GeV}$ & $2.8 \mathrm{GeV}$ & $1.22 \mathrm{GeV}$ \\
\hline
\end{tabular}

Table 2: Parameter values for the Herwig55 Monte Carlo, obtained in a fit to OPAL data. The Monte Carlo variable names and tuned values are given; other parameters were left at their default settings.

\begin{tabular}{|c|c|c|c|c|c|c|}
\hline \multicolumn{7}{|c|}{ Jetset73-Peterson parameter values } \\
\hline \hline MSTJ(11) & PARJ(21) & PARJ(41) & PARJ(42) & PARJ(54) & PARJ(55) & PARJ(81) \\
\hline 3 & $0.36 \mathrm{GeV}$ & 0.11 & $0.46 \mathrm{GeV}^{-2}$ & -0.046 & -0.0057 & $0.31 \mathrm{GeV}$ \\
\hline
\end{tabular}

Table 3: Parameter values for the Jetset73-Peterson Monte Carlo, obtained in a fit to OPAL data. The Monte Carlo variable names and tuned values are given; other parameters were left at their default settings.

\begin{tabular}{|c|c|c|c|c|c|}
\hline \multicolumn{7}{|c|}{ Normal-mixture selection } \\
\hline \hline & Jetset73 & Herwig55 & Cojets623 & Cojets612 & Jetset73-Peterson \\
\hline High energy jet & $97.0 \pm 0.2$ & $96.1 \pm 0.2$ & $97.1 \pm 0.2$ & $97.1 \pm 0.2$ & $97.0 \pm 0.1$ \\
Low energy jets & $51.5 \pm 0.3$ & $52.0 \pm 0.4$ & $51.5 \pm 0.5$ & $51.4 \pm 0.5$ & $51.5 \pm 0.2$ \\
\hline \hline \multicolumn{7}{|c|}{ Tagged selection } \\
\hline \hline \multicolumn{7}{|c|}{ Jetset73 } & Herwig55 & Cojets623 & Cojets612 & Jetset73-Peterson \\
\hline High energy jet & $96.8 \pm 0.7$ & $94.0 \pm 1.4$ & $96.1 \pm 1.2$ & $96.9 \pm 1.0$ & $96.8 \pm 0.6$ \\
Gluon-tagged jet & $16.4 \pm 1.5$ & $23.2 \pm 2.5$ & $16.2 \pm 2.3$ & $18.5 \pm 2.3$ & $20.0 \pm 1.3$ \\
Jet with vertex & $86.9 \pm 1.4$ & $82.8 \pm 2.2$ & $87.6 \pm 2.1$ & $84.6 \pm 2.1$ & $83.2 \pm 1.2$ \\
\hline Tagged event rate & $5.5 \pm 0.2$ & $3.9 \pm 0.2$ & $4.4 \pm 0.3$ & $4.9 \pm 0.3$ & $5.0 \pm 0.2$ \\
\hline
\end{tabular}

Table 4: Quark jet purity, $q$, in percent, for the jets of the normal-mixture and tagged data samples, as determined using different QCD event generators which include simulation of the detector. The tagged event rate is also shown, defined by the percentage of events from the normal-mixture sample which are contained in the tagged one. 


\section{Figure Captions}

Figure 1. Secondary vertex decay length distribution for the symmetric three-jet events. There are two entries per event, one for each of the two lower energy jets. The distribution is normalized by the total number of events, $N$, in the sample. The histogram shows the result from the Jetset-Peterson Monte Carlo, as explained in the text. For the Monte Carlo distribution, the contributions of bottom quark, charm quark, light quark and gluon jets are shown separately, by the shaded curves. (a) The decay length distribution from -0.4 to $0.6 \mathrm{~cm}$; (b) an expanded view around the interval used to tag quark jets.

Figure 2. (a) The ratio of the rate at which electrons and muons with momenta larger than $4 \mathrm{GeV} / c$ are observed in the highest energy (H.E.) jets of the tagged sample, to the corresponding quantity from the normal-mixture sample, displayed versus differential bins of the transverse momentum, $p_{T}$, of the leptons with respect to the jet axis; the last bin includes all $p_{T}$ values above $1.2 \mathrm{GeV} / c$. (b) The ratio of the rate at which secondary vertices are observed in the highest energy jets of the tagged sample, to the corresponding quantity from the normal-mixture sample, displayed versus differential bins of the decay length, L; the last bin includes all L values above $0.45 \mathrm{~cm}$. The hatched bands in parts (a) and (b) show the Monte Carlo predictions, with their statistical uncertainties.

Figure 3. (a) The mean particle energy value $\langle E\rangle$ versus the azimuthal angle $\psi$ in the three-jet event plane. The points with errors show the gluon jet side of the tagged sample. The histogram shows the average of the two event sides from the normal-mixture sample. (b) Monte Carlo distributions for the gluon jet side of events selected using the tagged (points with errors) and normal-mixture (histogram) criteria. For the normal-mixture sample, the quark and gluon jets are identified using the Monte Carlo information, as explained in the text.

Figure 4. (a) Inclusive scaled particle energy distributions of the gluon-tagged (g.tag) and normal-mixture (n.mix) jets; (b) ratio of the two curves in (a).

Figure 5. (a) Multiplicity distributions of the gluon-tagged and normal-mixture jets, using charged tracks and unassociated clusters; (b) same as (a), for charged tracks alone.

Figure 6. The fraction of the jet visible energy contained within a cone of half angle $10^{\circ}$ around the jet axis, for the gluon-tagged and normal-mixture data.

Figure 7. (a) The mean value of the fractional jet energy contained within a cone of half angle $\theta,\left\langle f_{E}(\theta)\right\rangle$, versus $\theta$, for the gluon-tagged and normal-mixture jets; (b) the fractional difference between the curves in $(\mathrm{a}), d_{E}(\theta)$, versus $\theta$.

Figure 8. (a) The differential distribution of the data shown in fig. $7(\mathrm{a}),\left(1 / E_{\text {vis. }}^{\text {jet }}\right) \mathrm{d} E_{\text {vis. }}^{\text {jet }} / \mathrm{d} \theta$, versus $\theta$, for the gluon-tagged and normal-mixture jets; (b) the fractional difference 
between the curves in (a), $d_{E}^{\text {diff. }}$, versus $\theta$.

Figure 9. The mean particle energy distribution, $\langle E\rangle$ versus $\psi$, after correction for the quark and gluon jet misidentification. The corrections are not valid in the region of the highest energy jets, shown by the hatched areas. No correction is made for detector acceptance and resolution. The Monte Carlo curves include full detector simulation and the same selection criteria as the normal-mixture data, except the quark and gluon jets are identified using the Monte Carlo information. The errors shown are statistical only. (a) The corrected quark jet distribution, (b) the corrected gluon jet distribution.

Figure 10. (a) Corrected inclusive scaled particle energy distributions of the gluon and quark jets; (b) ratio of the two curves in (a).

Figure 11. (a) and (b): Corrected multiplicity distributions of the quark and gluon jets, using charged tracks and unassociated clusters; (c) and (d): same as (a) and (b), for charged tracks alone.

Figure 12. (a) and (b): The corrected fraction of the quark and gluon jet visible energy contained within a cone of half angle $10^{\circ}$ around the jet axis.

Figure 13. (a) The corrected mean value of the fractional jet energy contained within a cone of half angle $\theta,\left\langle f_{E}(\theta)\right\rangle$, versus $\theta$, for the quark and gluon jets; $(b)$ the fractional difference between the curves in $(\mathrm{a}), d_{E}(\theta)$, versus $\theta$.

Figure 14. (a) The differential distribution of the data shown in fig. 13 (a), $\left(1 / E_{\text {vis. }}^{\text {jet }}\right) \mathrm{d} E_{\text {vis. }}^{\text {jet }} / \mathrm{d} \theta$, versus $\theta$, for the quark and gluon jets; (b) the fractional difference between the curves in (a), $d_{E}^{\text {diff. }}$, versus $\theta$. 
Figure 1
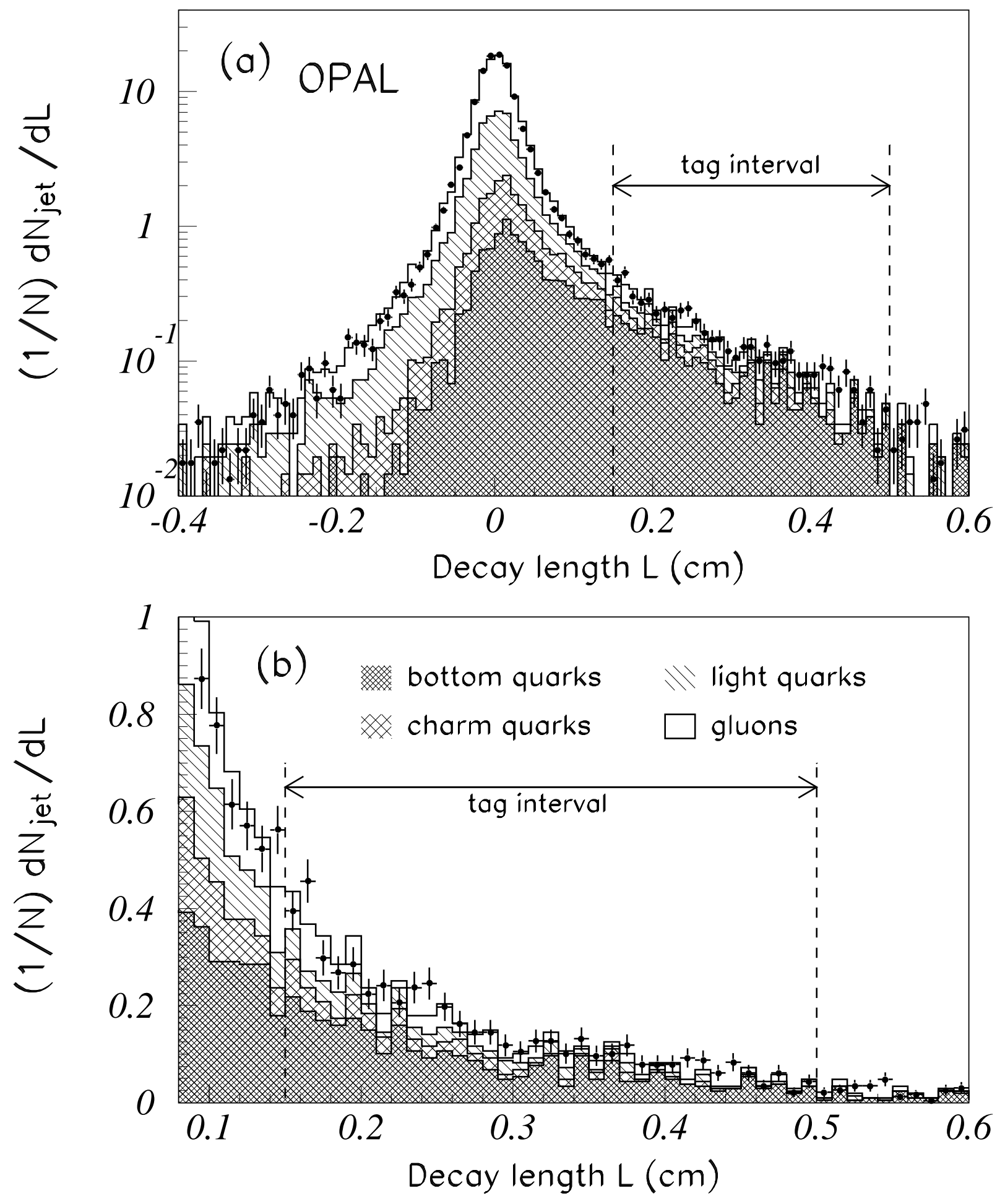
Figure 2
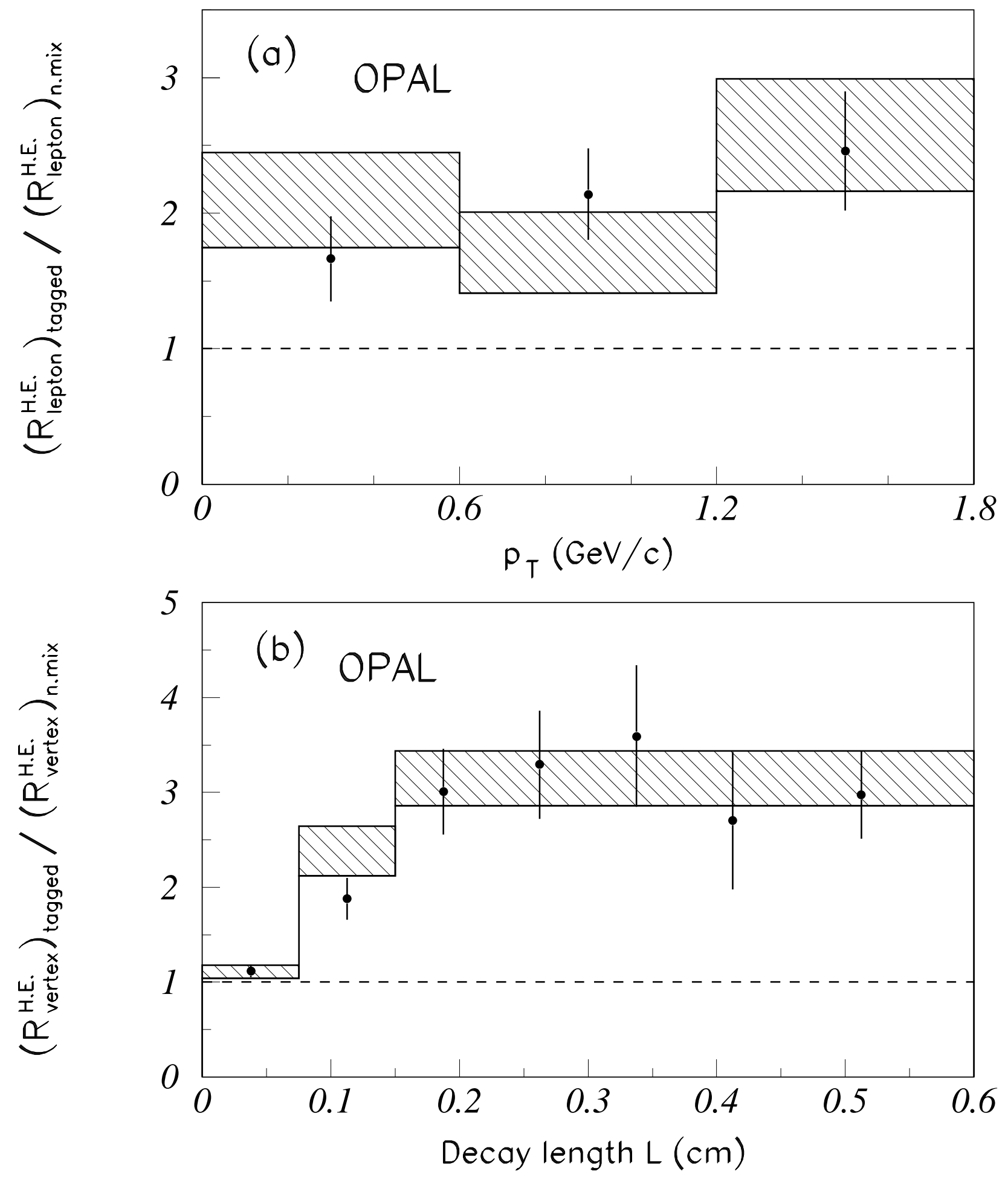
Figure 3
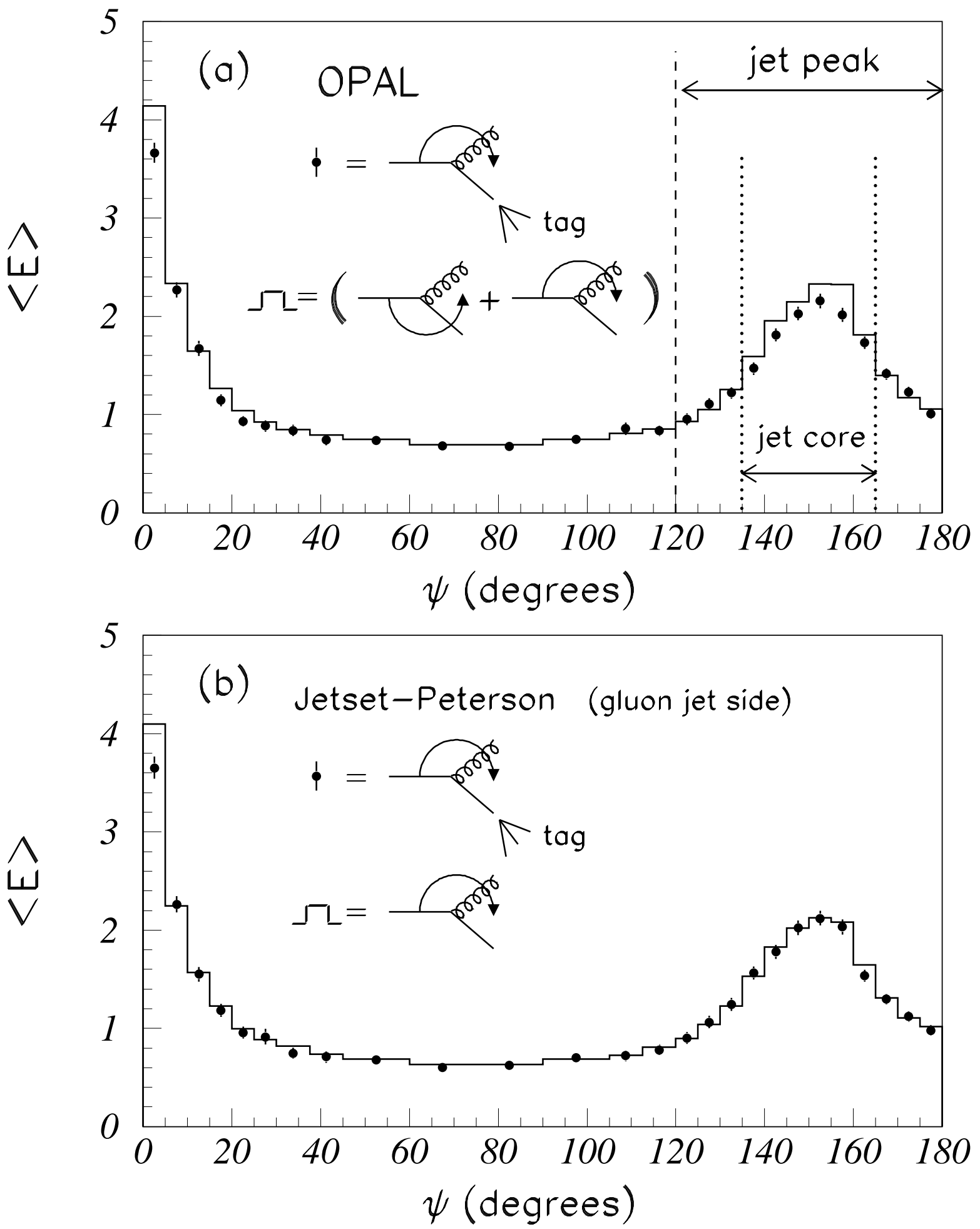
Figure 4
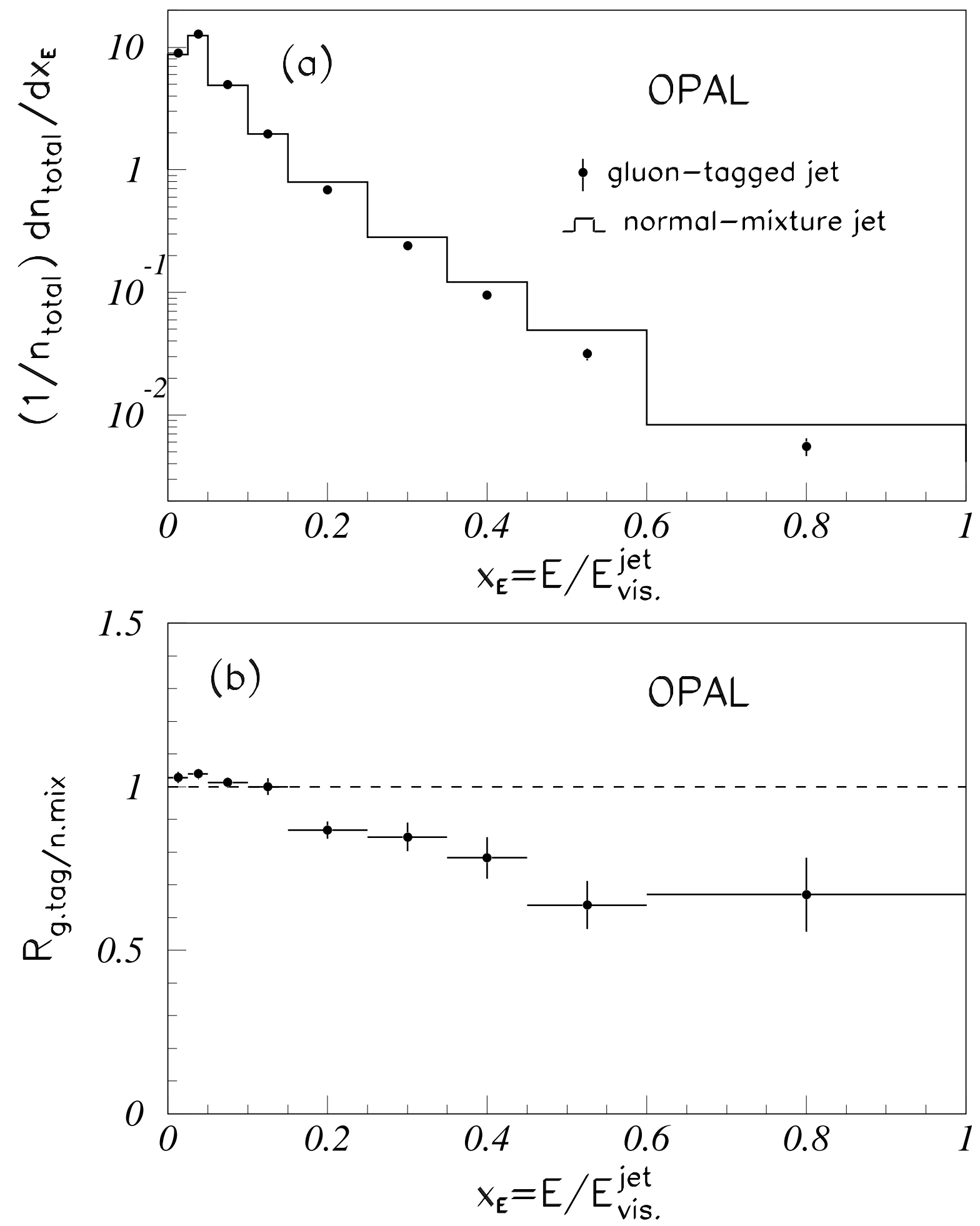
Figure 5
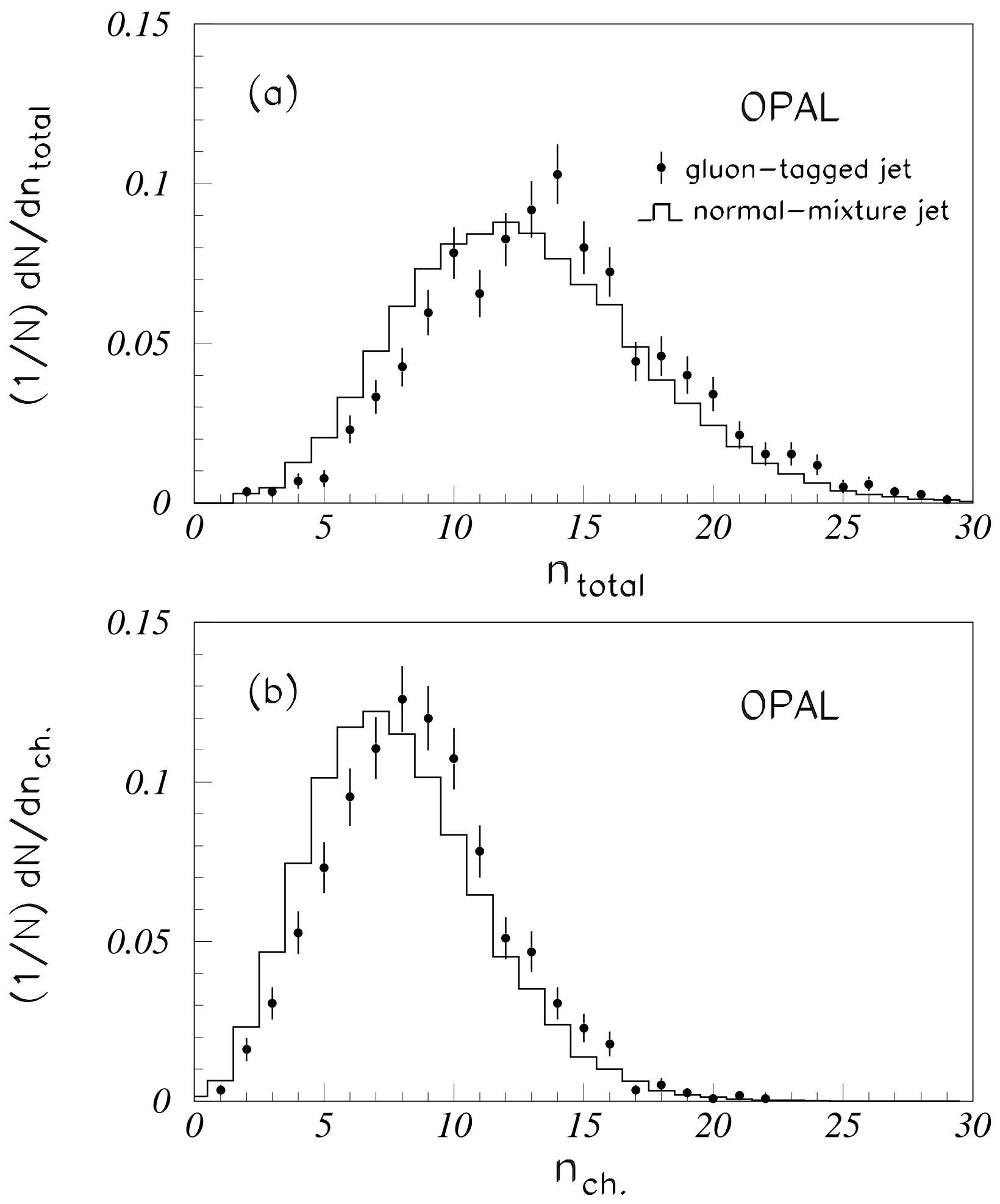
Figure 6

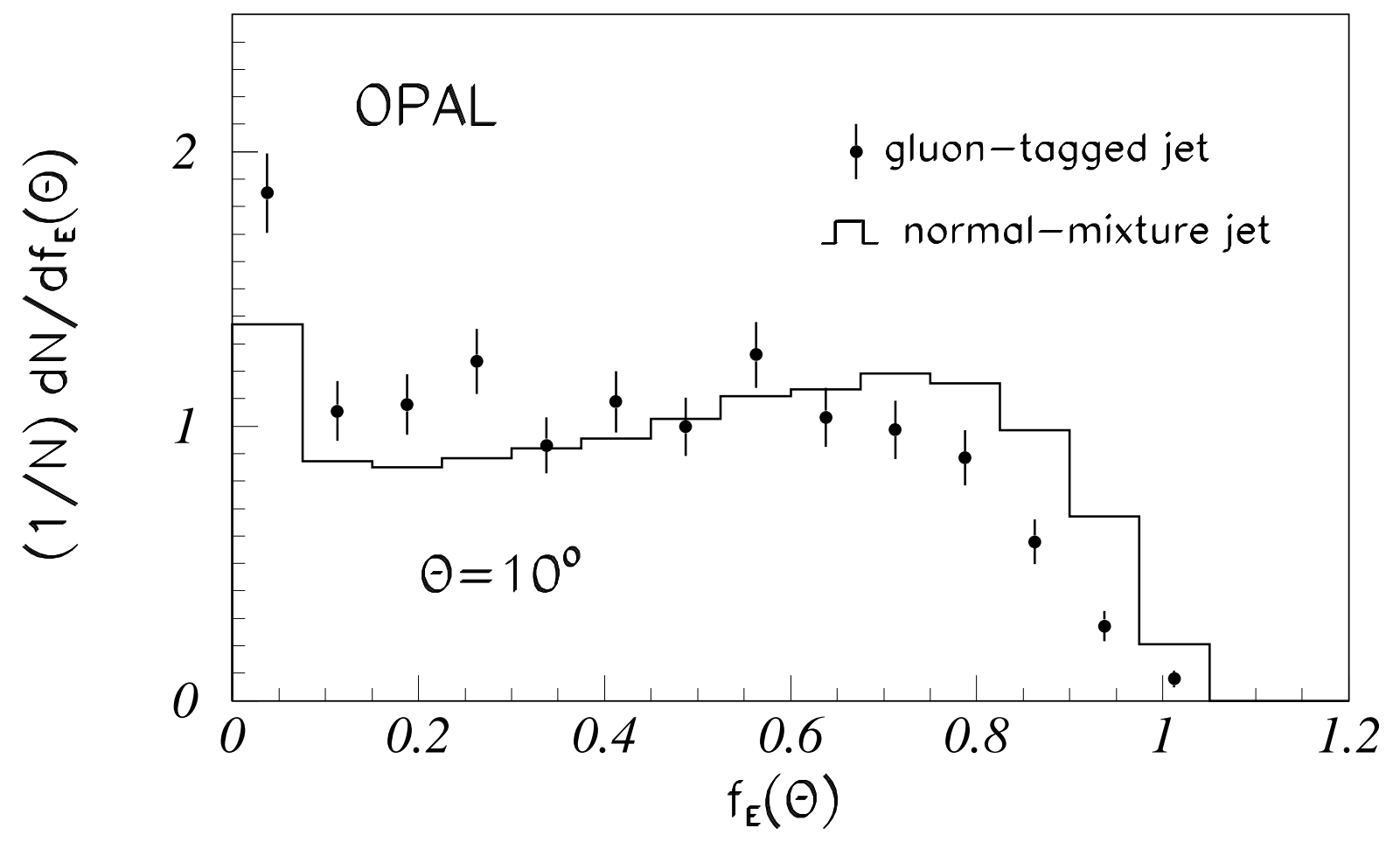


Figure 7

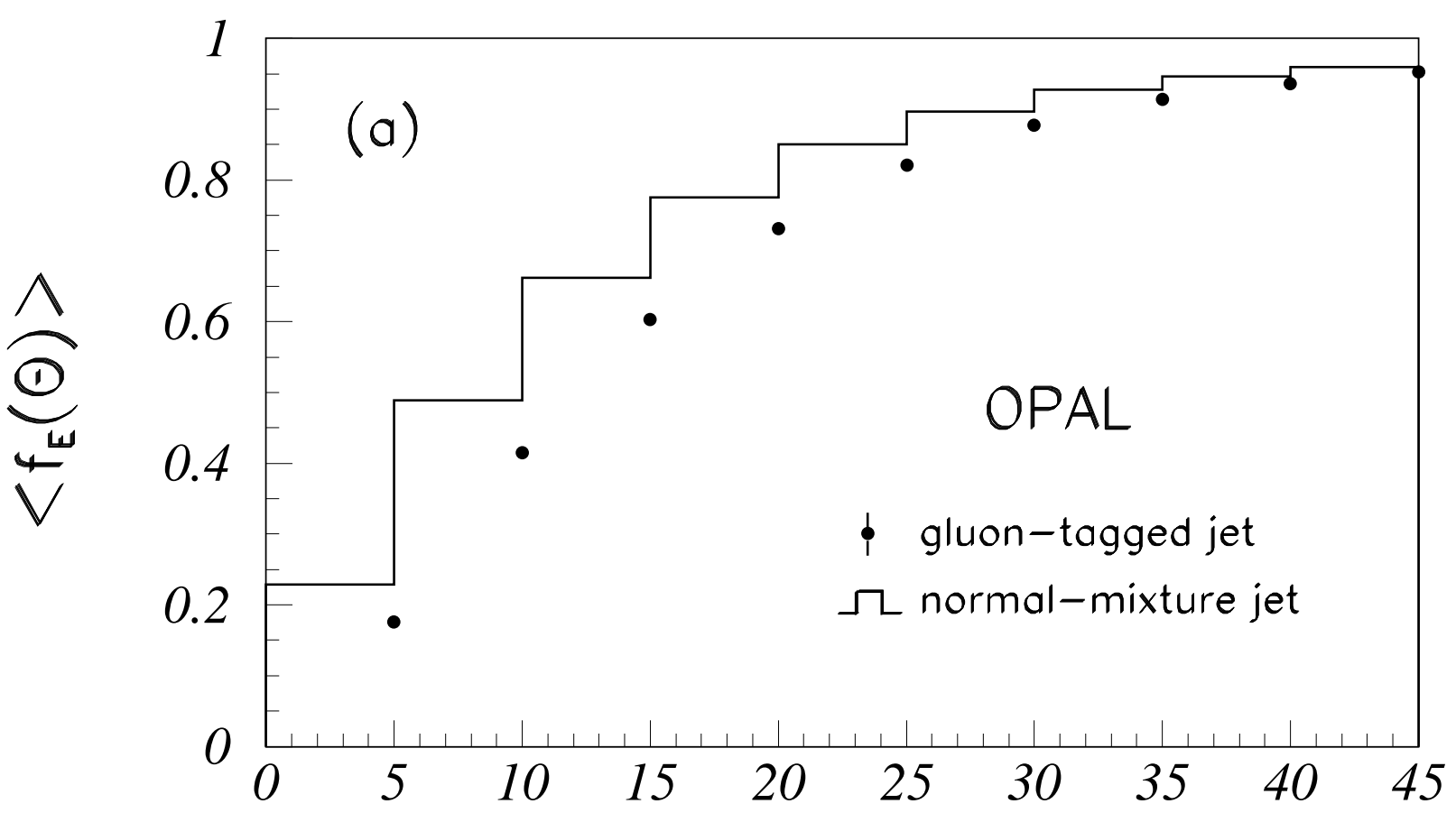

$\Theta$

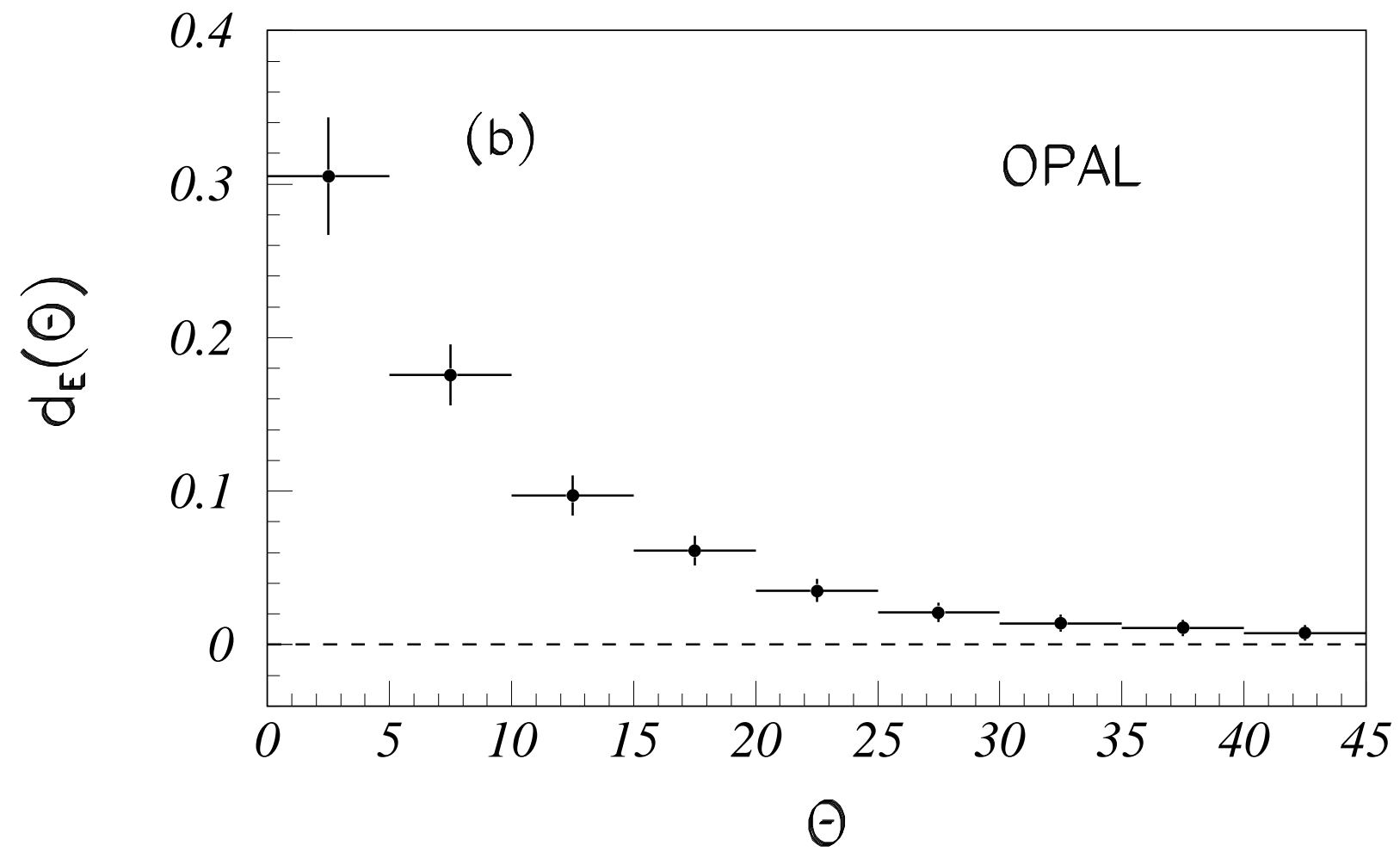


Figure 8
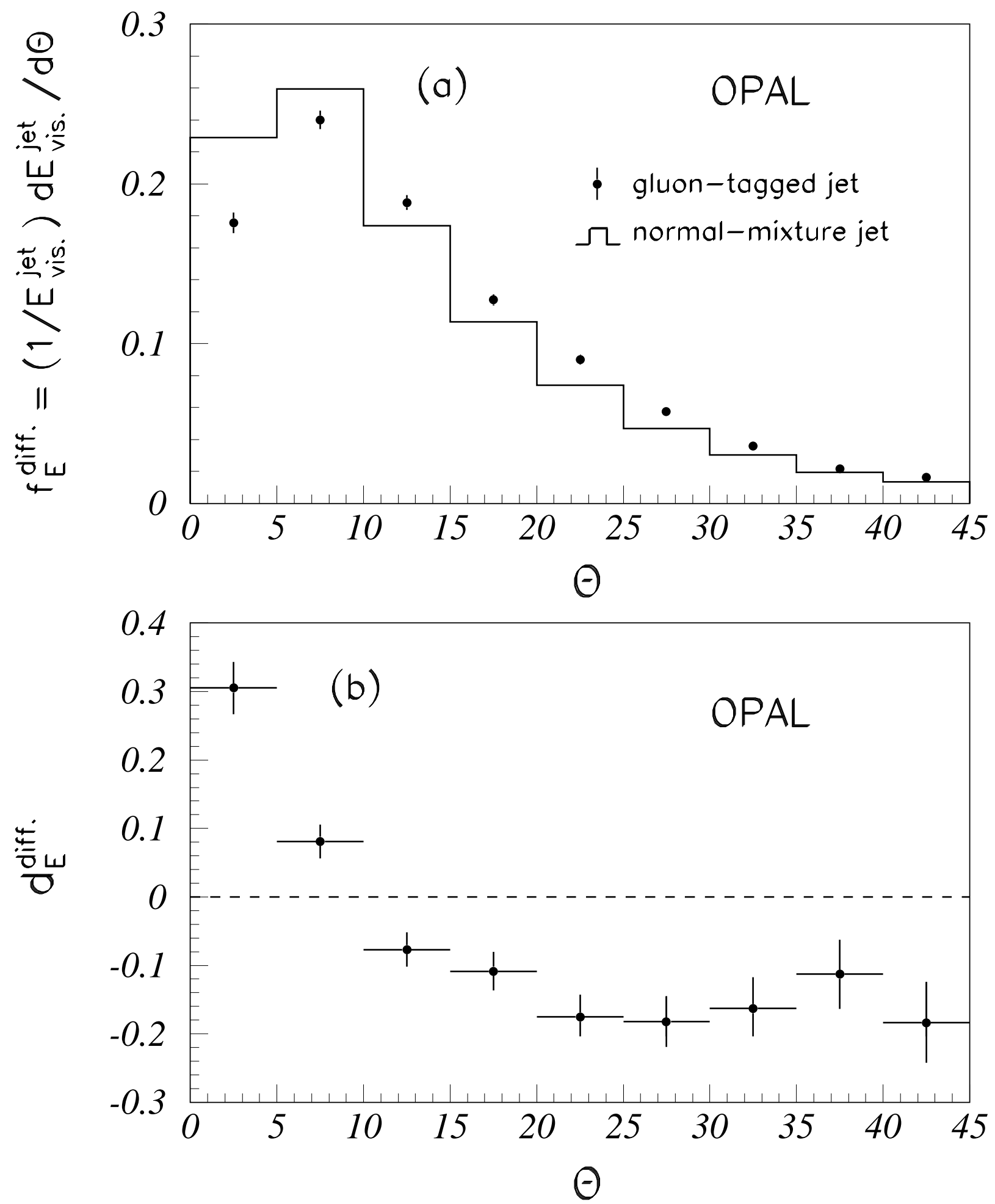
Figure 9
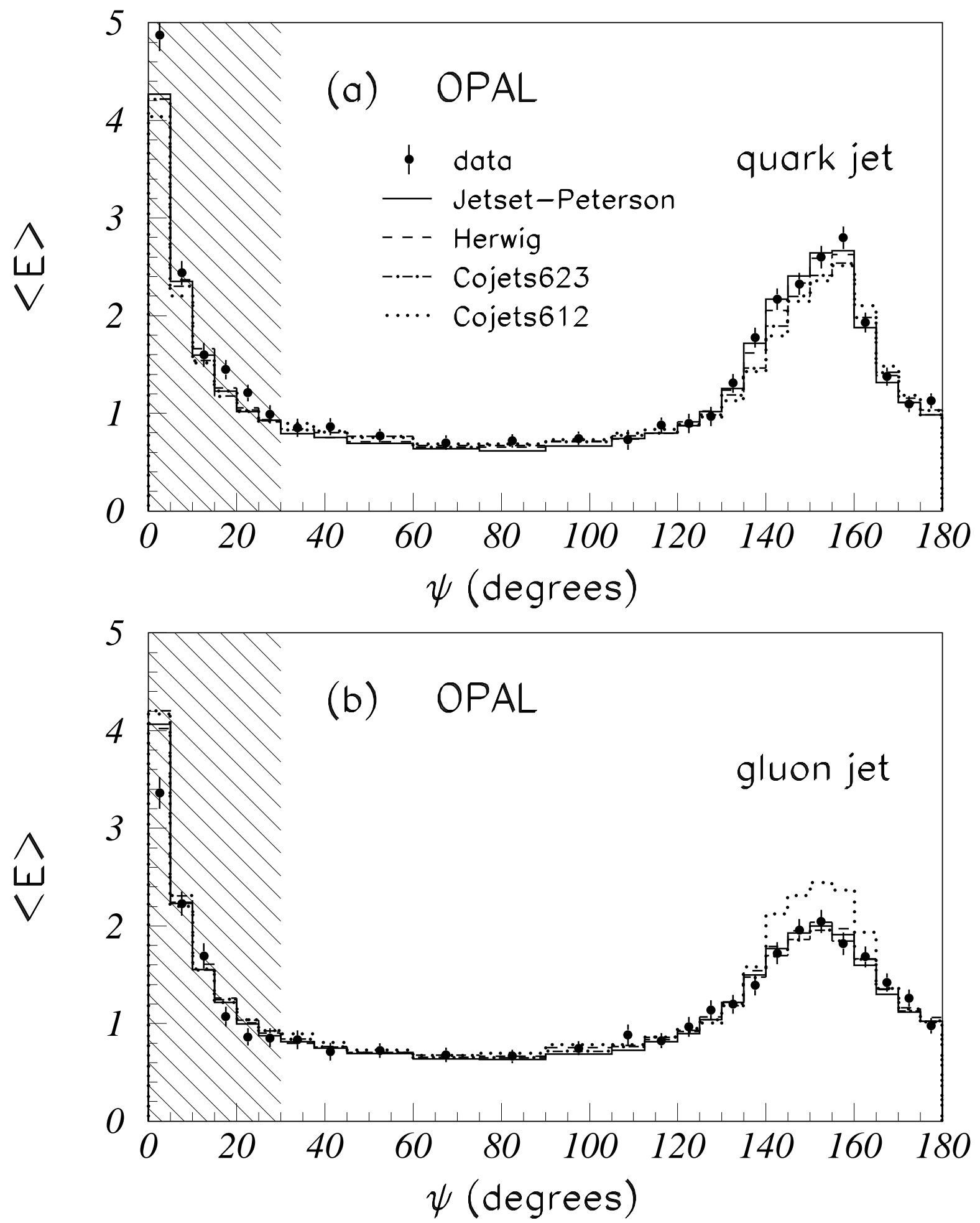
Figure 10
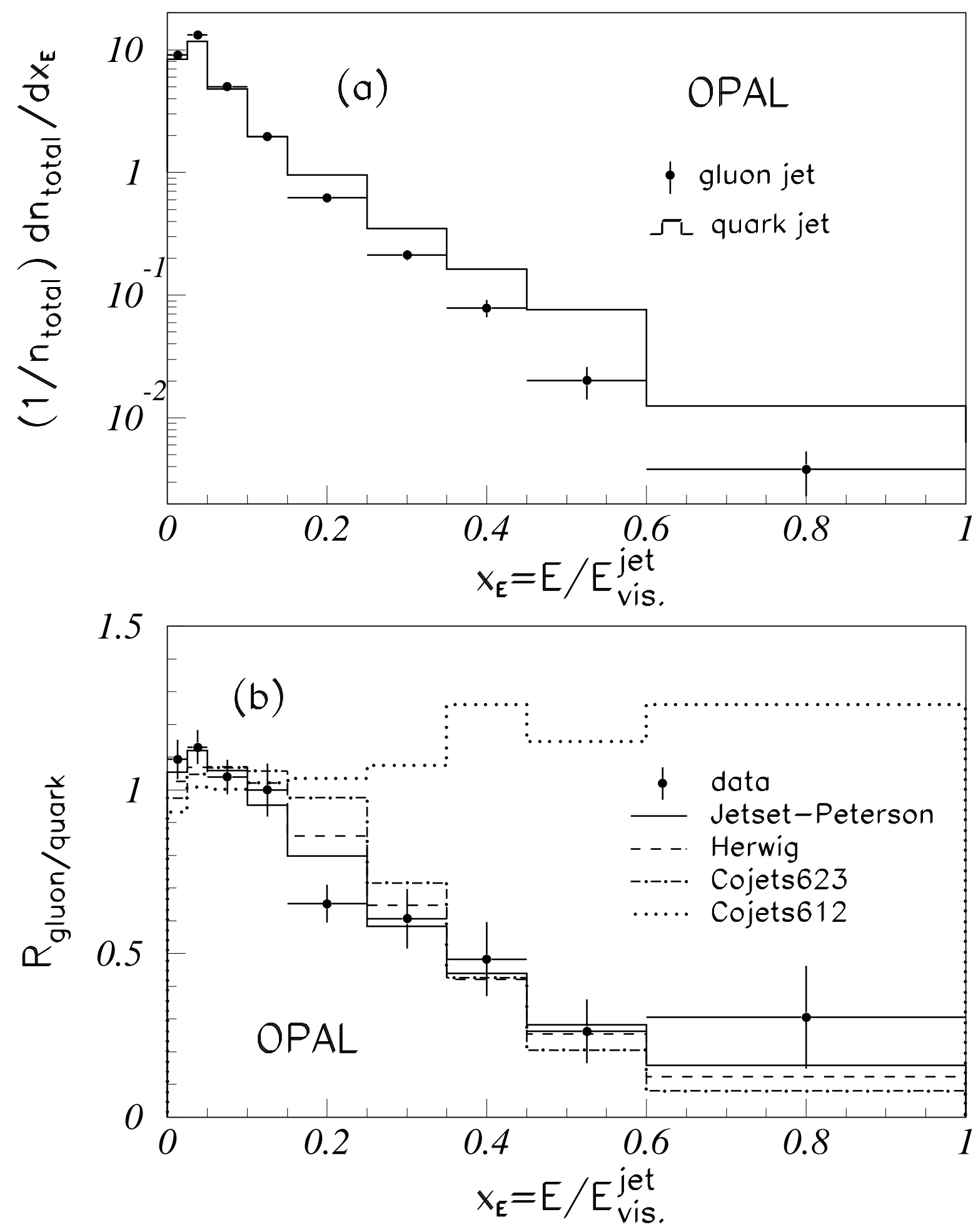
Figure 11
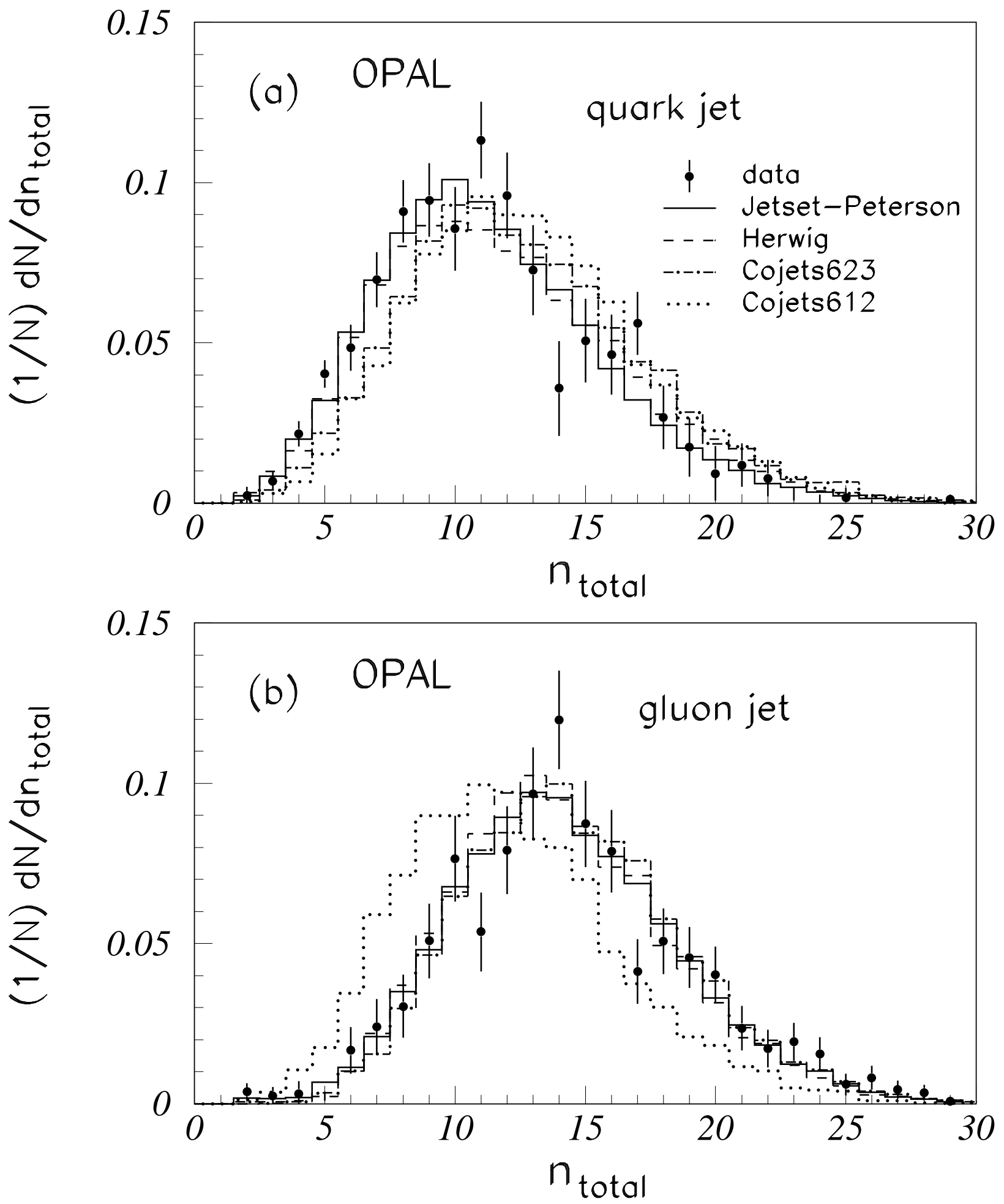
Figure 11
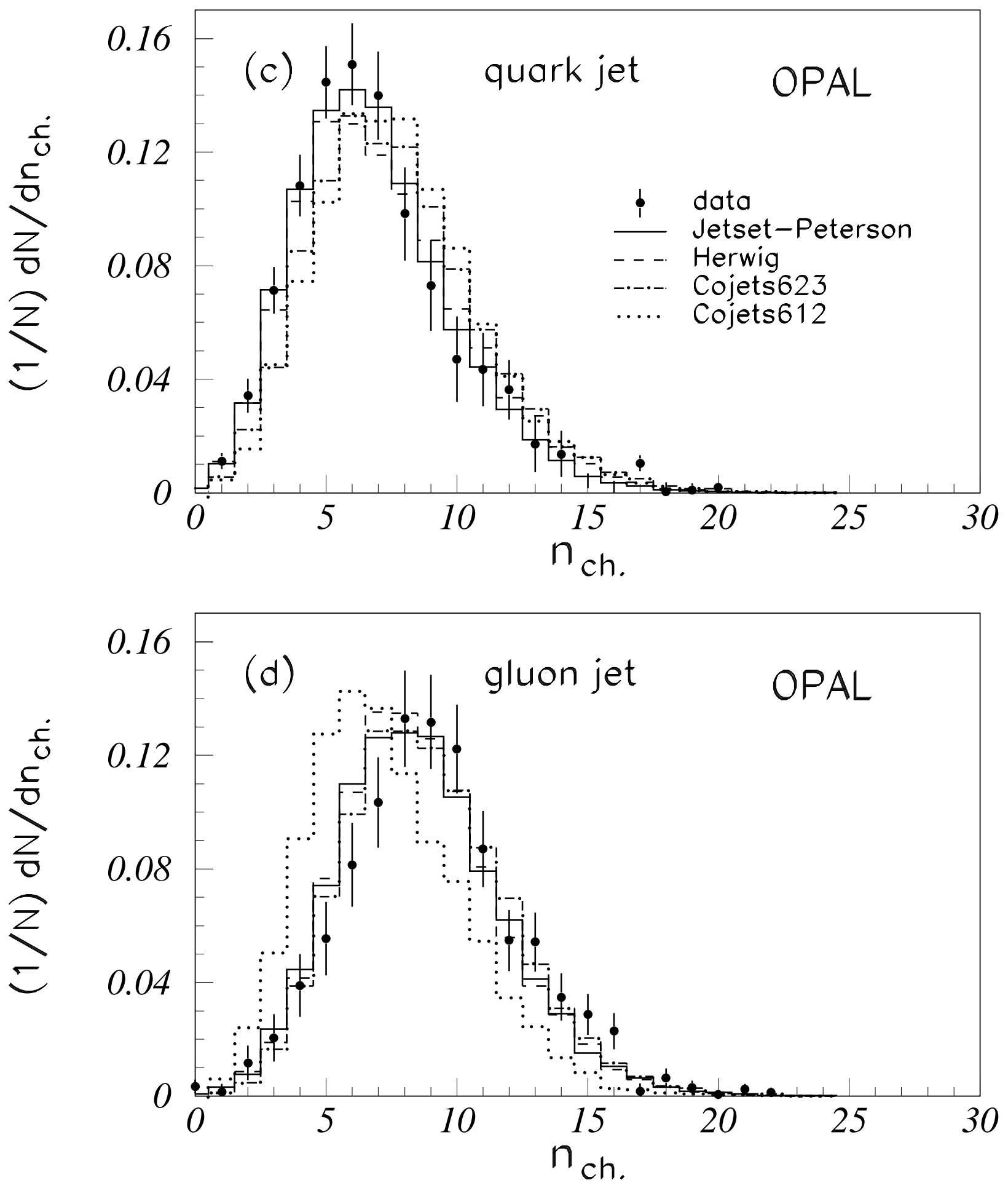
Figure 12
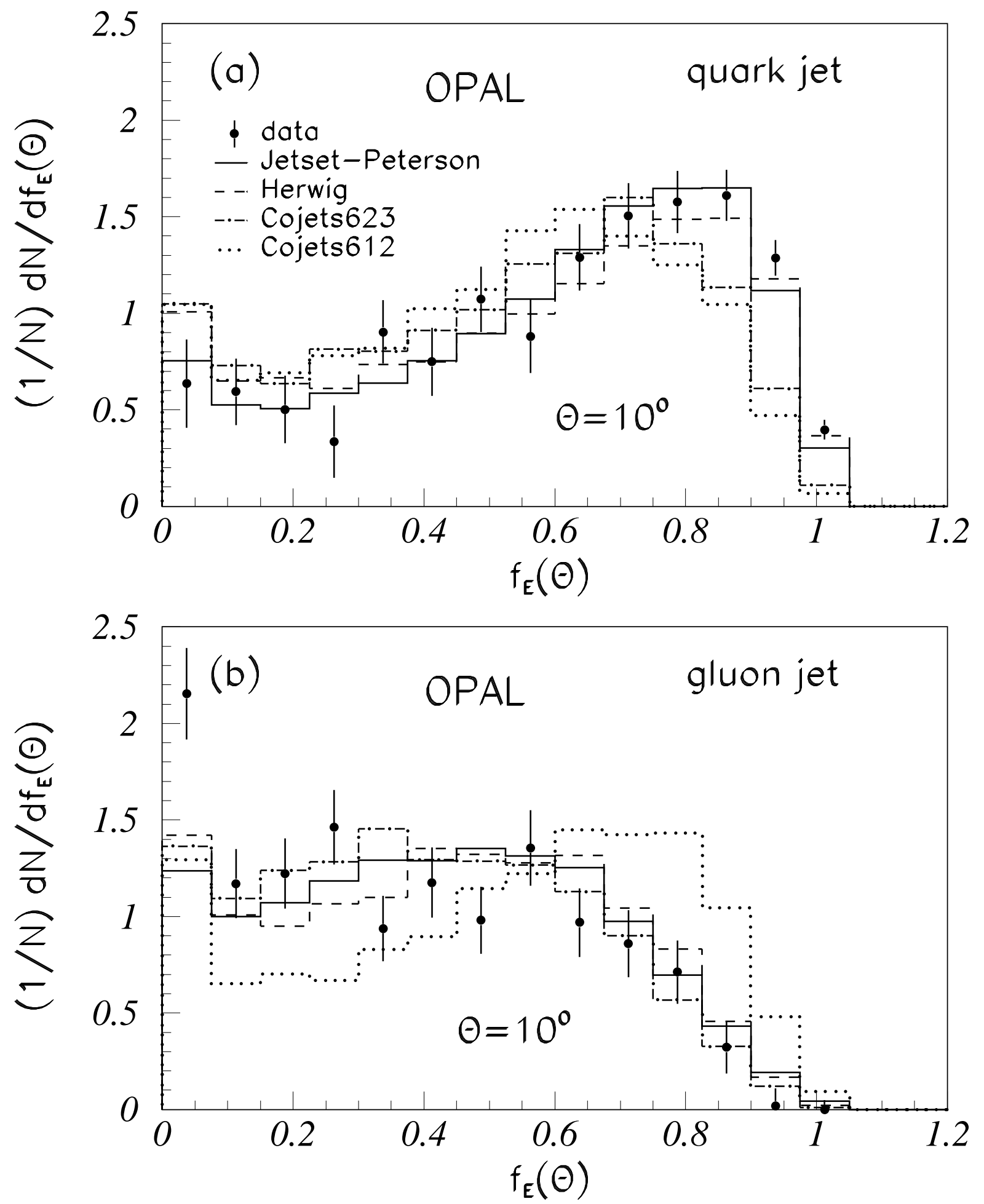
Figure 13
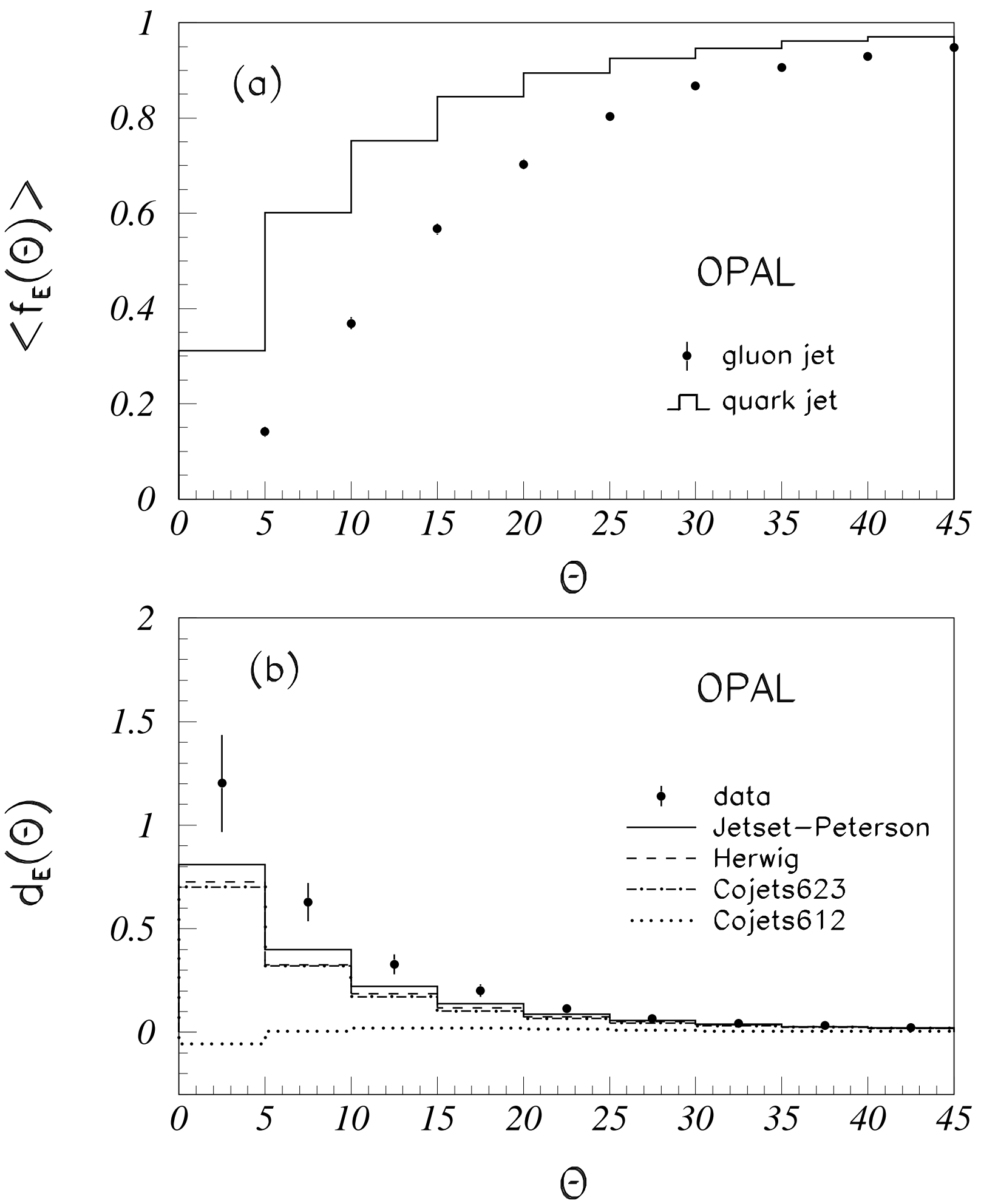
Figure 14
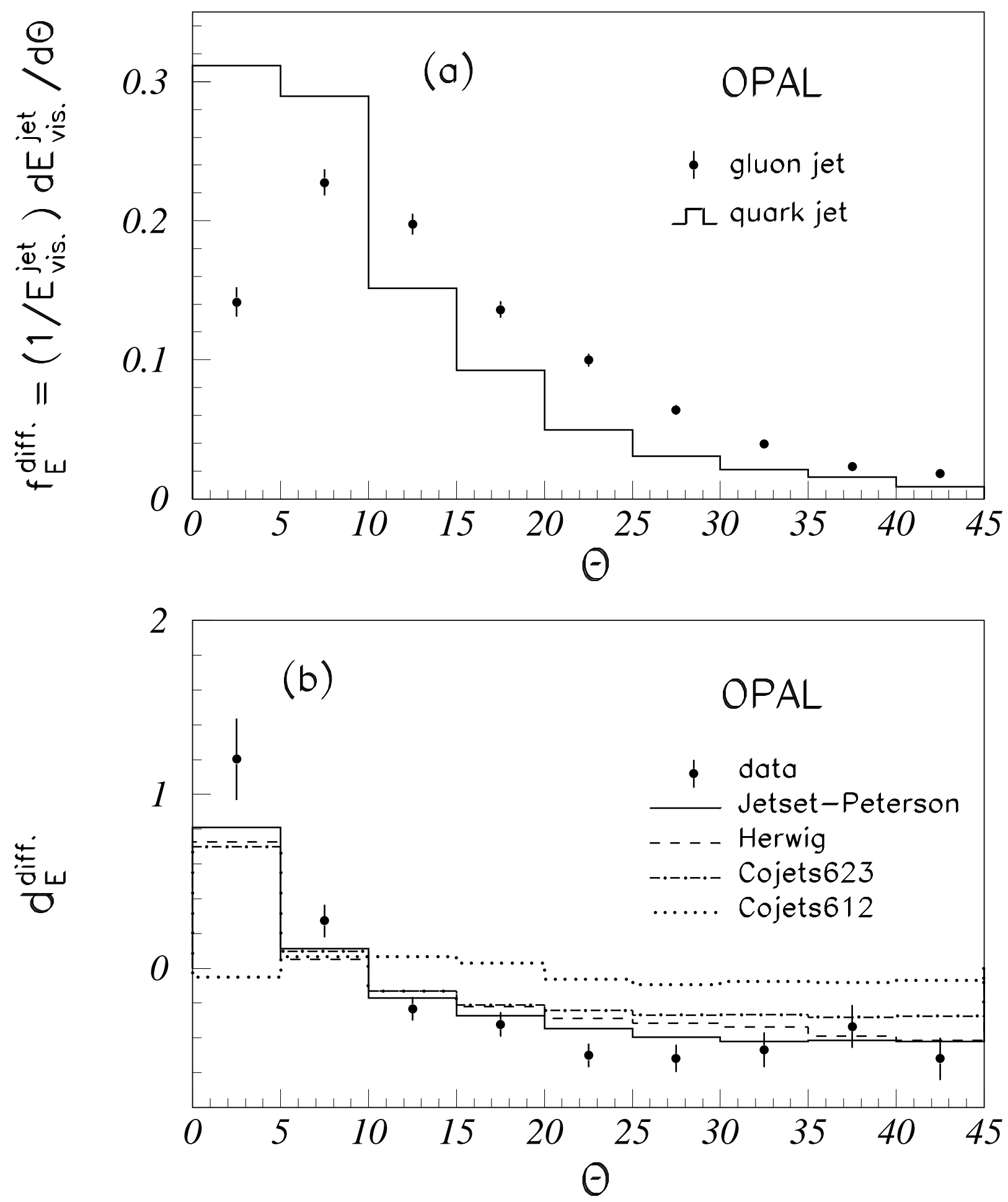